\title{
Mitochondrial stress response triggered by defects in protein synthesis quality control
}

\author{
Uwe Richter ${ }^{1, \star} \mathbb{D}$, Kah Ying $\mathrm{Ng}^{1, \star} \mathbb{\infty}$, Fumi Suomi ${ }^{1, \star}$, Paula Marttinen ${ }^{1}$, Taina Turunen ${ }^{1}$, Christopher Jackson ${ }^{2}$,

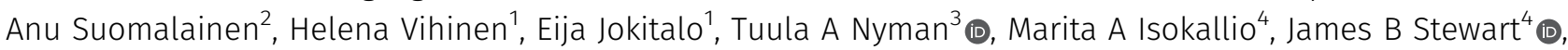 \\ Cecilia Mancini ${ }^{5}$, Alfredo Brusco ${ }^{5}$ (D), Sara Seneca ${ }^{6}$, Anne Lombès ${ }^{7} \mathbb{B}$, Robert W Taylor ${ }^{8}$, Brendan J Battersby ${ }^{1}$ (1)
}

\begin{abstract}
Mitochondria have a compartmentalized gene expression system dedicated to the synthesis of membrane proteins essential for oxidative phosphorylation. Responsive quality control mechanisms are needed to ensure that aberrant protein synthesis does not disrupt mitochondrial function. Pathogenic mutations that impede the function of the mitochondrial matrix quality control protease complex composed of AFG3L2 and paraplegin cause a multifaceted clinical syndrome. At the cell and molecular level, defects to this quality control complex are defined by impairment to mitochondrial form and function. Here, we establish the etiology of these phenotypes. We show how disruptions to the quality control of mitochondrial protein synthesis trigger a sequential stress response characterized first by OMA1 activation followed by loss of mitochondrial ribosomes and by remodelling of mitochondrial inner membrane ultrastructure. Inhibiting mitochondrial protein synthesis with chloramphenicol completely blocks this stress response. Together, our data establish a mechanism linking major cell biological phenotypes of AFG3L2 pathogenesis and show how modulation of mitochondrial protein synthesis can exert a beneficial effect on organelle homeostasis.
\end{abstract}

DOI 10.26508/Isa.201800219 | Received 20 October 2018 | Revised 16 January 2019 | Accepted 17 January 2019 | Published online 25 January 2019

\section{Introduction}

Mitochondria contain a unique protein synthesis machinery devoted to the exclusive translation of a small number of proteins encoded in the mitochondrial genome (Ott et al, 2016). In humans, these number only 13 hydrophobic membrane proteins, which form core subunits of three respiratory chain complexes and the $F_{1} F_{O}$ ATP synthase required for oxidative phosphorylation. Assembly of these complexes requires an additional 80 structural proteins encoded in the nucleus and imported into mitochondria, so protein synthesis in the cytosol and mitochondria needs temporal and spatial coordination. On the mitochondrial side, protein synthesis must be tightly regulated with complex assembly or proteolytic quality control to prevent nascent chains from over-accumulating in the membrane.

Proteostasis within mitochondria requires a collection of compartmentalized chaperones and proteases that trace their evolutionary origin back to alphaproteobacteria (Quirós et al, 2015). One factor implicated in the quality control of mitochondrial protein synthesis is a membrane-anchored AAA (ATPases Associated with diverse cellular Activities) protease complex composed of AFG3L2 subunits. In humans, this hexameric complex affects the stability of newly synthesized mitochondrial proteins (Zurita Rendón \& Shoubridge, 2012; Hornig-Do et al, 2012; Richter et al, 2015). In the budding yeast, proteolytic maturation of the mitochondrial ribosomal protein Mrpl32 has been ascribed to this AAA protease complex, a step required for ribosome assembly (Nolden et al, 2005). In contrast, this mechanism is not observed in mammalian tissues (Almajan et al, 2012). It is worth noting that site-specific proteolytic processing of substrates appears to be incompatible with the conserved mechanistic function of AAA proteases, which couple ATP hydrolysis for protein unfolding with processive proteolysis of substrates into oligopeptides (10 amino acids) (Sauer \& Baker, 2011). Thus, the effect on ribosome biogenesis is possibly indirect, perhaps, reflecting a downstream response of mitochondrial dysfunction.

Pathogenic mutations that disrupt the AFG3L2 quality control complex are associated with human diseases: hereditary spastic paraplegia (HSP), spastic ataxia (SPAX5), spinocerebellar ataxia (SCA28), and progressive external ophthalmoplegia (PEO) (Casari et al, 1998; Di Bella et al, 2010; Pierson et al, 2011; Gorman et al, 2015). A hallmark of these diseases, also seen in mouse models (Almajan et al, 2012), is fragmentation of the mitochondrial membrane

\footnotetext{
${ }^{1}$ Institute of Biotechnology, University of Helsinki, Helsinki, Finland ${ }^{2}$ Research Programs Unit-Molecular Neurology, University of Helsinki, Helsinki, Finland ${ }^{3}$ Department of Immunology, Institute of Clinical Medicine, University of Oslo and Oslo University Hospital, Oslo, Norway ${ }^{4}$ Max Planck Institute for Biology of Ageing, Cologne, Germany ${ }^{5}$ Department of Medical Sciences, University of Torino, Torino, Italy ${ }^{6}$ Center for Medical Genetics/Research Center Reproduction and Genetics, Universitair Ziekenhuis Brussel, Brussels, Belgium ${ }^{7}$ Faculté de médecine Cochin, Institut Cochin Institut national de la santé et de la recherche médicale U1016, Centre national de la recherche scientifique Unités Mixtes de Recherche 8104, Université Paris 5, Paris, France ${ }^{8}$ Wellcome Centre for Mitochondrial Research, Institute of Neuroscience, Newcastle University, Newcastle upon Tyne, UK
} 
morphology, loss of mitochondrial ribosomes, and defective oxidative phosphorylation. Fragmentation of mitochondrial morphology prevents axonal trafficking in neurons (Kondadi et al, 2014) and is considered a key cell biological event in the molecular pathogenesis of these disorders (Ferreirinha et al, 2004). Nonetheless, the etiology of these three distinguishing phenotypes has remained unknown for the last $20 \mathrm{y}$.

AFG3L2 dysfunction is known to activate the metalloprotease OMA1, triggering processing of the dynamin-related GTPase OPA1 tethered to the inner membrane releasing it into a soluble pool (Ehses et al, 2009). This activation mechanism appears to be independent of the membrane potential (Ehses et al, 2009). Under these circumstances, mitochondrial morphology is remodelled from a reticular shape to a fragmented swollen state. Preventing stressinduced OPA1 processing has been shown to be beneficial in a mouse model of neurodegeneration (Korwitz et al, 2016). However, no mechanism has been established, so far, that could reconcile AFG3L2 quality control to OPA1 processing and dynamic changes in membrane morphology.

In this study, we report that impaired quality control of mitochondrial nascent chain synthesis triggers a novel stress response first characterized by OMA1 activation and then remodelling of membrane ultrastructure from the reduction in mitochondrial ribosomes. Together, our data establish a mechanism linking major cell biological phenotypes of AFG3L2 pathogenesis and show how modulation of mitochondrial protein synthesis can exert a beneficial effect on organelle homeostasis.

\section{Results}

\section{A mitochondrial stress response from impaired nascent chain quality control}

AFG3L2 dysfunction activates OMA1, inducing proteolysis of the long OPA1 forms (membrane tethered) independently of alterations to membrane potential (Ehses et al, 2009; Kondadi et al, 2014) (Fig 1A-C). Recently, we showed how AFG3L2 modulated a mitochondrial membrane proteotoxicity arising from the small molecule actinonin and characterized by OMA1 activation (Richter et al, 2013, 2015). The magnitude of the drug response was proportional to the rate of translation elongation on mitochondrial ribosomes and/or the abundance of AFG3L2. A subsequent study demonstrated actinonin specifically bound to the AFG3L2 orthologue in Plasmodium falciparum and Toxoplasma gondii (Amberg-Johnson et al, 2017), suggesting a conserved mechanism across the eukaryotic tree. Therefore, we asked whether the OMA1 activation associated with AFG3L2 defects was triggered by stress arising from mitochondrial protein synthesis. AFG3L2 siRNA leads to proteolytic cleavage of long OPA1 and mitochondrial fragmentation (Fig 1B and D). Both phenotypes were robustly blocked by inhibiting protein synthesis on mitochondrial ribosomes with chloramphenicol (Fig 1B and D).

Another prominent phenotype observed with AFG3L2 dysfunction in genetically modified mouse models is reduction in the abundance of mitochondrial ribosomes (Nolden et al, 2005; Almajan et al, 2012). After $5 \mathrm{~d}$ of AFG3L2 siRNA knockdown in human cultured fibroblasts, there was no difference on the abundance of the mitochondrial ribosomal protein $\mathrm{uL} 11 \mathrm{~m}$ (Fig 1B). This suggests the ribosomal phenotype may not be direct but rather a response to the progressive loss of this AAA protease complex. To test the idea, we investigated the consequences from a longer AFG3L2 siRNA knockdown by waiting an additional three days (no extra siRNA was added) before collecting the cells. The rationale for this approach is that AFG3L2 is a core fitness gene (Hart et al, 2015), which impedes the use of genome editing to make a genetic knockout in cultured human cells. After $8 \mathrm{~d}$ of culture, we still observed a robust reduction of AFG3L2 and OPA1 processing, which is now accompanied with a decrease in mitochondrial ribosomal proteins from both the large and small subunit (Fig 1B). Inhibition of mitochondrial protein synthesis with chloramphenicol completely suppressed not only OPA1 processing but also the reduction of mitochondrial ribosomes (Fig 1B). Together, the data indicate AFG3L2 dysfunction generated a proteotoxicity that arose specifically from newly synthesized mitochondrial proteins, triggering an organelle stress response.

Because the mitochondrial genome (mtDNA) encodes both ribosomal RNAs (rRNAs) essential for mitochondrial ribosome assembly, we sought to distinguish whether the loss of the mitochondrial ribosomes was simply a downstream response from a reduced mtDNA copy number. OPA1 abundance is known to affect the copy number and integrity of the mitochondrial genome (mtDNA) (Hudson et al, 2008). We used Southern blotting to quantify the mitochondrial copy number and genome integrity. No differences were observed with either short- or long-term AFG3L2 siRNA knockdown (Fig 1E and F).

Prohibitins form a scaffold in the inner membrane that physically interacts with and regulates the AFG3L2 complex (Steglich et al, 1999; Nolden et al, 2005). Loss of the prohibitin scaffold also triggers OMA1-dependent processing of OPA1 (Merkwirth et al, 2008). To test if mitochondrial nascent chain proteotoxicity was the OMA1 trigger, we used siRNA against prohibitin 2 (PHB2) and treated cells with chloramphenicol. Complete inhibition of mitochondrial protein synthesis with chloramphenicol blocked the OPA1 processing (Fig S1A). In contrast, loss of the other mitochondrial inner membrane-bound AAA protease, YME1L, or its scaffold STOML2 (Wai et al, 2016) did not induce a translationdependent OPA1 processing (Fig S1B and C). This points to a primary importance of the AFG3L2 complex for the quality control of nascent chains emerging from mitochondrial ribosomes in the matrix.

Next, we addressed the relevance of the stress response with a pathogenic mutation in AFG3L2. MEFs heterozygous for a knock-in mutation M665R (M666R in humans) associated with spinocerebellar ataxia 28 (SCA28) (Cagnoli et al, 2010; Mancini et al, 2018) did not exhibit stress-activated OPA1 proteolysis or reduction of mitochondrial ribosomal proteins at the steady state (Fig 1G). This finding is consistent with observations from a number of human heterozygous pathogenic mutations in AFG3L2 (Di Bella et al, 2010). In contrast, MEFs homozygous for the M665R mutation exhibited mitochondrial translation-dependent OPA1 processing at the steady state (Fig 1G).

Temperature is a well-known modulator of protein folding, so we used heat shock as a stress during mitochondrial protein synthesis to test whether it is a modifier of the M665R Afg3l2 mutation. Heat shock triggered mitochondrial translation-dependent OPA1 processing and reduction of mitochondrial ribosomal proteins 
A

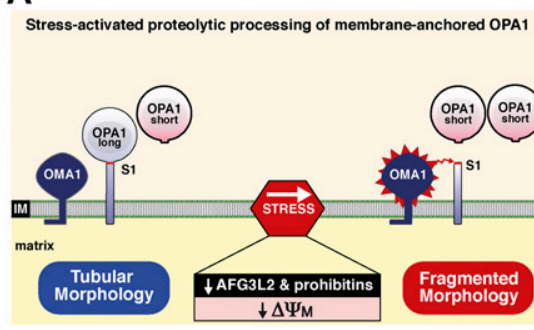

C

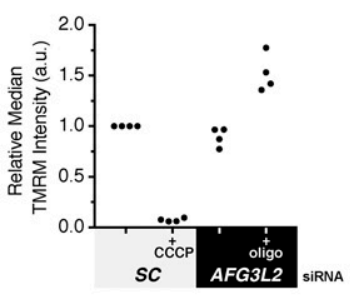

D

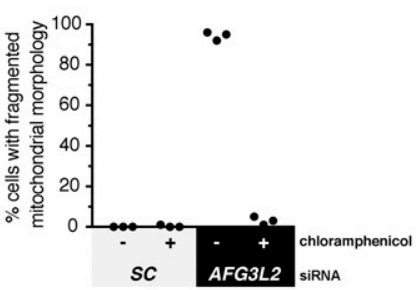

$\mathbf{F}$
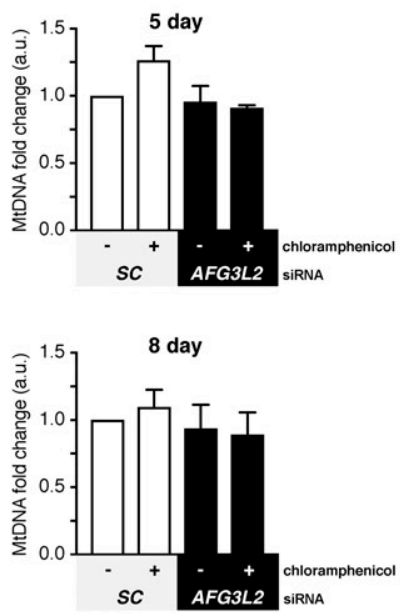

B
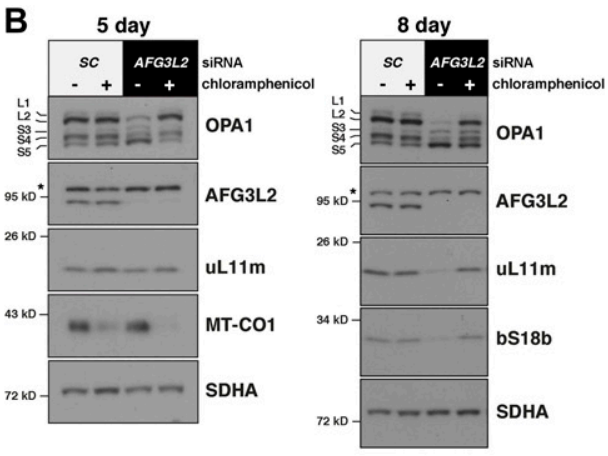

E

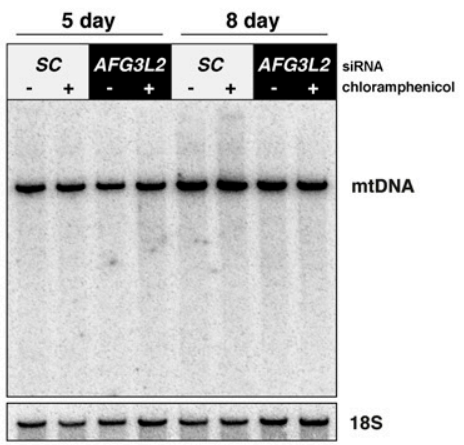

G

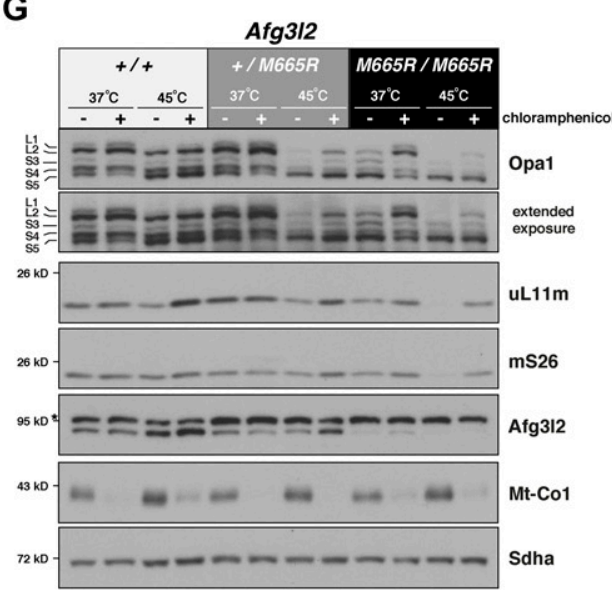

Figure 1. A mitochondrial translation stress response regulating OPA1 and ribosome homeostasis. (A) Diagram illustrating the stress-induced regulated proteolysis of membrane-tethered OPA1 by the OMA1 metalloprotease. (B) Immunoblotting of whole-cell lysates of human fibroblasts treated with the indicated siRNAs with (+) and without (-) chloramphenicol (CA). *Nonspecific band detected with the AFG3L2 antibody. (C) Scatter plot of mitochondrial membrane potential analyzed by tetramethylrhodamine (TMRM) staining of HEK293 cells treated with the indicated siRNAs from independent experiments. CCCP is a positive control to dissipate the membrane potential. Oligomycin (oligo) inhibits the F1FO ATP synthase, which leads to hyperpolarization unless ATP hydrolysis is required to maintain the membrane potential. (D) Scatter plot quantification of mitochondrial morphology under light microscopy in human fibroblasts treated with the indicated siRNAs with or without CA. Data are from three independent experiments, $n=100$. (E) Southern blot of human fibroblasts treated with the indicated siRNAs with (+) and without (-) CA. (F) Quantification of Southern blotting in (E) from three independent experiments. Data represent mean \pm SD. (G) Immunoblotting of mouse embryonic fibroblasts with the indicated $A f g 3 l 2$ genotypes grown at $37^{\circ} \mathrm{C}$ or heat shocked for $4 \mathrm{~h}$ at $45^{\circ} \mathrm{C}$ with (+) and without (-) CA. IM, inner membrane; S1, OMA1 proteolytic site; SC, scrambled. proportional to the allelic copy number of the pathogenic M665R Afg312 mutation (Fig 1G). This effect was not observed in the wildtype MEFs. To investigate the robustness of the finding in further detail, we used heat shock in a series of control cell lines (wild-type human immortalized fibroblasts, myoblasts, HEK293, and an additional MEF line) for up to $4 \mathrm{~h}$. None of these human or mouse cultured cell lines exhibited stress-activated OPA1 processing with heat shock (Figs S1C-E and S2D and G). Thus, heat shock appeared to elicit a mitochondrial translation-dependent stress response only with pathogenic mutations in AFG3L2. Collectively, our data demonstrate that AFG3L2 has a primary role in regulating proteotoxicity that arises during protein synthesis on mitochondrial ribosomes. Absence of this quality control step activates a conserved progressive mitochondrial stress response initiated on the inner membrane.

\section{Aberrant mitochondrial mRNA triggers a translation-dependent stress response}

Next, we set out to investigate the source of the proteotoxicity that arises during nascent chain synthesis on mitochondrial ribosomes. Proteotoxicity could be generated by the impaired quality control of all 13 proteins, a subset or specific nascent chains, or a type/class of genetic mutation. Currently, it is not possible to modify open 
reading frames within the human mitochondrial genome with gene editing, so we turned to genetic mutations linked to human mitochondrial disorders that disrupt the synthesis or stability of the 13 mitochondrial proteins.

Our first goal was to test whether unstable nascent chains triggered OMA1 activation. We took several approaches to investigate this question, focusing on critical subunits required for the assembly of individual oxidative phosphorylation complexes. Assembly defects to the $F_{1} F_{0}$ ATP synthase generate instability of MT-ATP6 and MT-ATP8 nascent chains (Fig S2A and B). We tested a mild and severe disruption to complex assembly (Čižková et al, 2008; Rak et al, 2011), but neither induced OMA1 activation (Fig S2C). Next, we turned to the two catalytic core subunits of cytochrome c oxidase: MT-CO1 and MT-CO2. COX10 is an essential enzyme involved in the biosynthetic pathway for heme $a$, a critical metal moiety for cytochrome c oxidase assembly and required for stability of MT-CO1 nascent chains (Antonicka et al, 2003). The absence of MT-CO1 also disrupts MT-CO2 stability (Fig S2D). Genetic disruptions to COX10 function, however, did not activate OMA1 even after heat shock (Fig S2E). These data suggest that instability of four of the 13 nascent chains does not act as proteotoxic trigger.

To expand this line of inquiry, we asked whether a severe disruption to the synthesis and stability of all 13 nascent chains could be an OMA1 trigger. To test this question, we used human myoblasts homoplasmic for the m.8344A>G mutation in the mitochondrial tRNA ${ }^{\text {Lys }}$ linked to MERRF (myoclonus epilepsy, ragged-red fibers) (Boulet et al, 1992; Sasarman et al, 2008). In these cells, there is a severe defect to the synthesis and stability of all mitochondrial nascent chains (Fig S2F and G) (Richter et al, 2018). However, we observed no stress-induced OMA1 activation at the steady-state or even following a 4-h heat shock (Fig S2G). Collectively, our data rule out the instability of mitochondrial nascent chains as the proteotoxic trigger for OMA1 activation.

Most human pathogenic mtDNA mutations occur within tRNA genes and tend to be very rare in the protein-coding regions (Taylor \& Turnbull, 2005; Yarham et al, 2010). An exception is MT-ATP6, which is an essential subunit of the $F_{1} F_{O}$ ATP synthase (Zhou et al, 2015). We investigated whether mutations in this open reading frame could induce the mitochondrial translation-dependent stress response. In this collection of patient fibroblasts, there are three different missense mutations that generate apparently stable proteins at steady state, one nonsense mutation that leads to reduced synthesis of the full-length MT-ATP6, and an MT-ATP6 mRNA lacking a stop codon (Fig 2A and B) (Seneca et al, 1996; Auré et al, 2013; Jackson et al, 2017). At steady state, none of these pathogenic mutations displayed stress-induced OPA1 processing
A

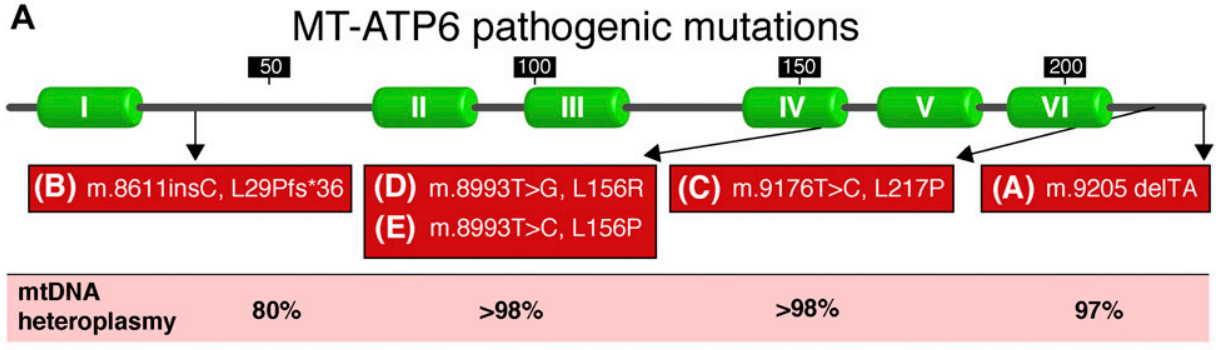

B

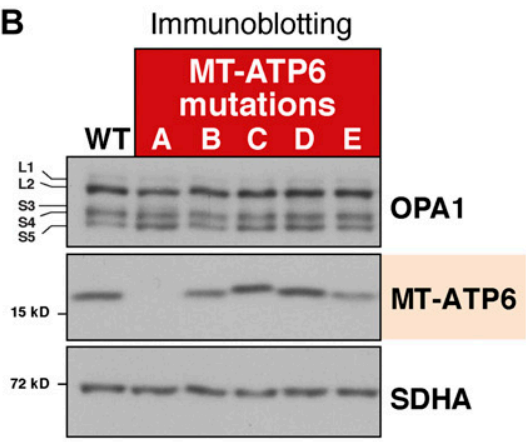

\section{C $\quad{ }^{35} \mathrm{~S}$ metabolic labeling}

\section{MT-ATP6} mutations

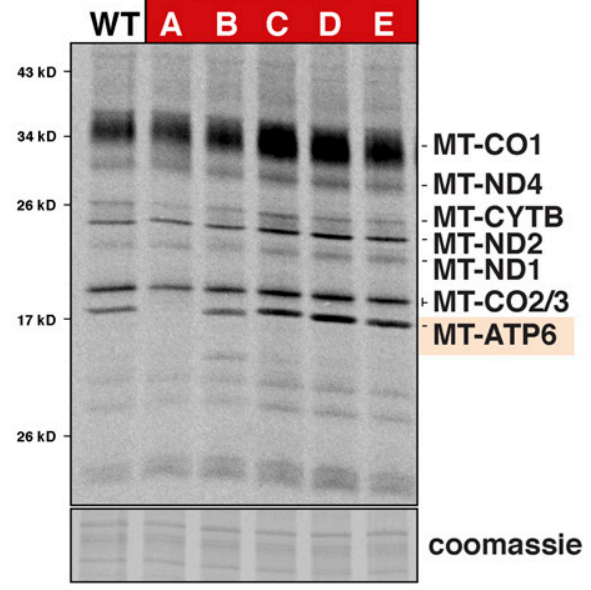

D

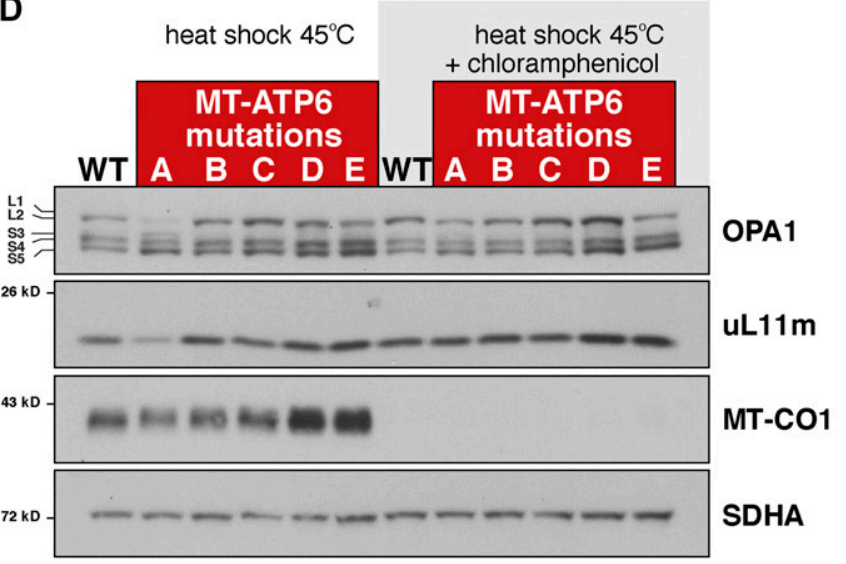

Figure 2. Heat shock during translation of an aberrant mitochondrial mRNA triggers a mitochondrial stress response.

(A) Diagram of human MT-ATP6 illustrating the location of pathogenic mutations in the protein and the heteroplasmy level of the mutations in patient fibroblasts. (B) Immunoblotting of whole-cell lysates from patient fibroblasts with the indicated MT-ATP6 mutations and a WT control. (C) A 30-min pulse metabolic labelling with ${ }^{35}$ S-methionine/cysteine of mitochondrial protein synthesis in patient fibroblasts with the indicated MT-ATP6 mutations. (D) Immunoblotting of whole-cell lysates from fibroblasts with the indicated MT-ATP6 mutations heat shocked for $4 \mathrm{~h}$ at $45^{\circ} \mathrm{C}$ with (+) and without (-) chloramphenicol. 
A

\section{MT-ATP6 m.9205delTA}

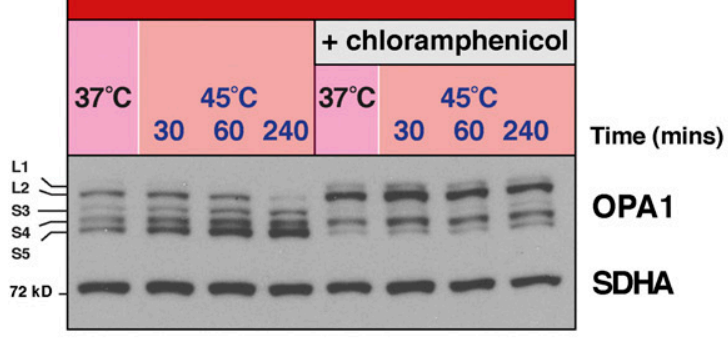

D
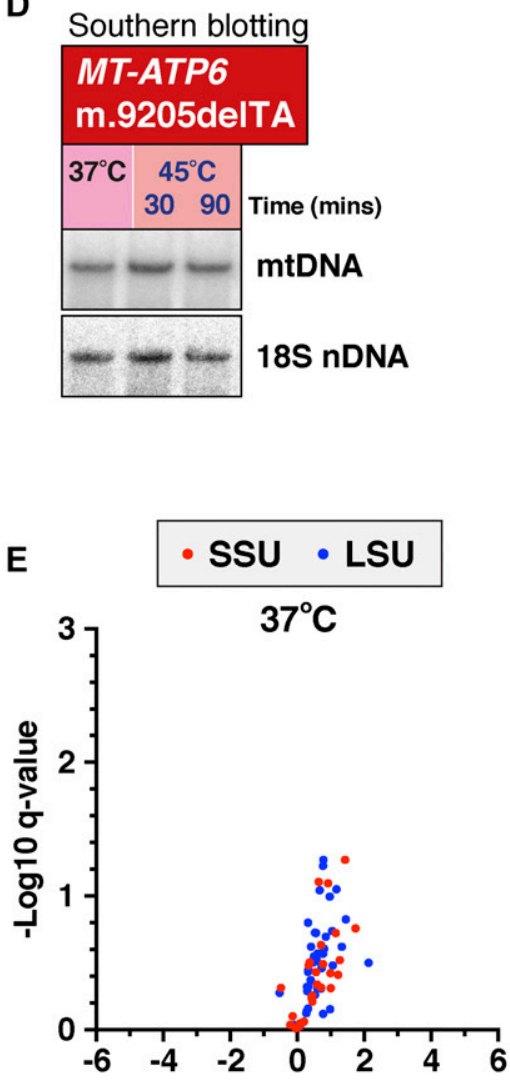

$\log _{2}$ F.C. MT-ATP6 delTA / control

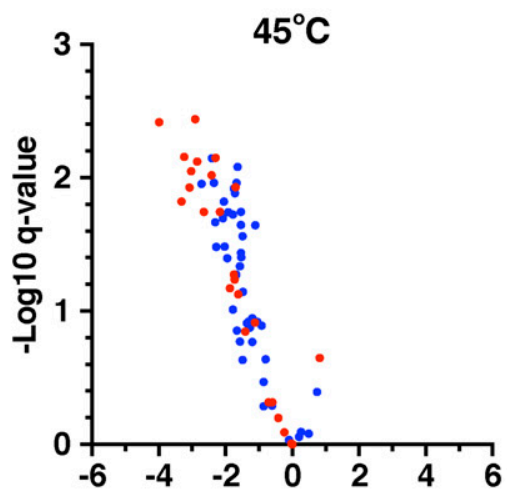

$\log _{2}$ F.C. MT-ATP6 deITA / control
B
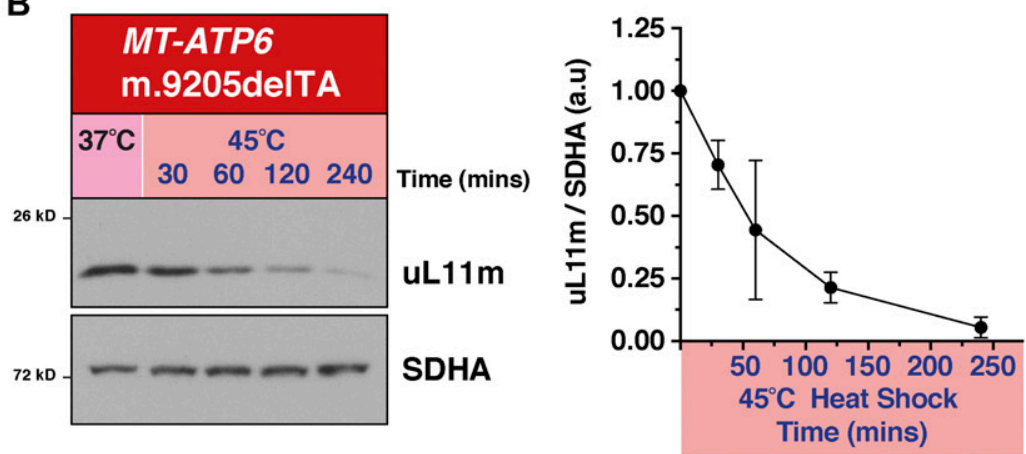

C

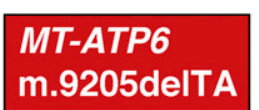

$37^{\circ} \mathrm{C} \quad 45^{\circ} \mathrm{C}$

$30 \quad 90$ Time (mins)

12S rRNA

16S rRNA

18S rRNA

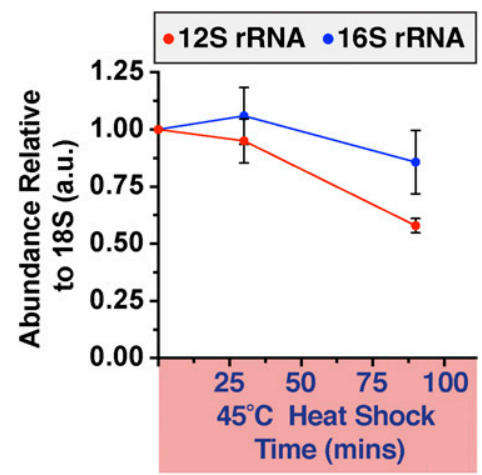

$\mathbf{F}$

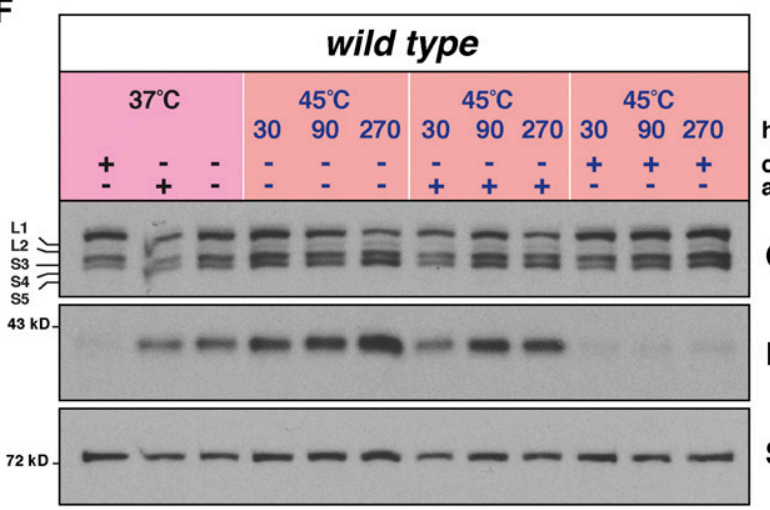

heat shock (mins)

chloramphenicol anisomycin

OPA1

MT-CO1

SDHA

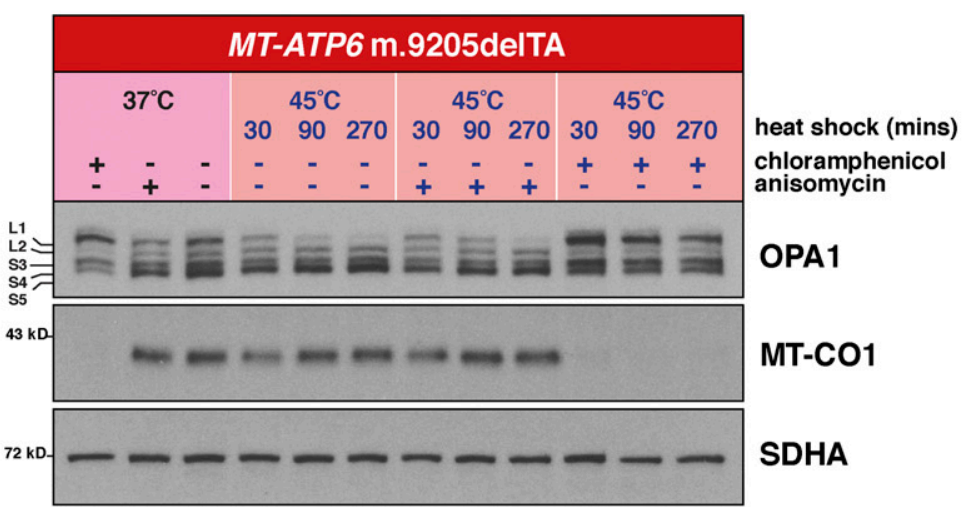


(Fig 2B). The non-stop MT-ATP6 mRNA (m.9205delTA), because of a 2-bp microdeletion, was associated with undetectable protein synthesis (Fig 2B and C). Using high-resolution respirometry, however, along with an oligomycin titration assay, which is a specific enzymatic inhibitor of the $F_{1} F_{0}$ ATP synthase, we could measure a residual level of a functional complex (Fig S3). Deep sequencing of the mitochondrial genomes in the MT-ATP6 m.9205delTA fibroblasts (Table S1) identified approximately 3\% wild-type mtDNA molecules, which is presumably the source for the functional MT-ATP6 that was undetectable by metabolic labelling or immunoblotting.

We asked whether heat shock could modulate any of the MTATP6 mutations and thereby activate the mitochondrial translation stress response. To our surprise, heat shock induced OPA1 proteolytic processing but only with the MT-ATP6 m.9205delTA mutation (Fig 2D). Heat shock also reduced the abundance of the mitochondrial ribosomal protein $\mathrm{LL} 11 \mathrm{~m}$ in these fibroblasts (Fig 2D). Both phenotypes could be completely blocked when mitochondrial protein synthesis was inhibited with chloramphenicol (Fig 2D). Together, the data suggested that only nascent chain synthesis from an aberrant mitochondrial mRNA during heat shock can activate the same stress response observed with AFG3L2 dysfunction.

Next, we sought to investigate in more detail the mitochondrial translation-dependent stress response with the MT-ATP6 m.9205delTA mutation. Heat shock induced time-dependent processing of OPA1 and reduction in UL11m (Fig 3A and B). Interestingly, there is a differential decay on the reduction in the mitochondrial rRNA, which appears to be faster for the 12S rRNA of the small subunit (Fig 3C). Importantly, this loss of mitochondrial rRNA and ribosomal protein is not due to alterations in the copy number of mtDNA (Fig 3D). To generate a more comprehensive and quantitative analysis of the changes to mitochondrial ribosomal protein abundance, we used label-free quantitative liquid chromatography-mass spectrometry/mass spectrometry. Using this method, we detected a significant and robust loss of mitochondrial ribosomal proteins from both the large and small subunits during heat shock in the MT-ATP6 m.9205delTA patient fibroblasts (Fig 3E). Assembly of the $F_{1} F_{O}$ ATP synthase requires subunits synthesized on both cytosolic and mitochondrial ribosomes. Thus, unbalanced accumulation of newly synthesized subunits of the complex from cytosolic ribosomes could trigger the response in the MT-ATP6 m.9205delTA patient fibroblasts during heat shock. To test this idea, we inhibited cytoplasmic protein synthesis with anisomycin, a well-known inhibitor of the $805 \mathrm{sy-}$ tosolic ribosome but did not observe any modulation of the OPA1 processing phenotype (Fig $3 \mathrm{~F}$ ). Therefore, heat shock during the translation of an aberrant mitochondrial mRNA triggers a ribosomal decay pathway and OMA1 activation.
To address the consequence of heat shock to mitochondrial protein synthesis, we pulse labelled mitochondrial proteins with ${ }^{35} \mathrm{~S}$ for 30 min at different time points during a 4-h incubation (Fig 4A). In the MT-ATP6 m.9205delTA fibroblasts, we observed a rapid attenuation of mitochondrial protein synthesis during heat shock (Fig $4 \mathrm{~B}$ ) that preceded the reduction in ribosomal proteins (Fig 3B). Because we showed AFG3L2 is integral to the quality control of mitochondrial nascent chain synthesis (Fig 1), we asked whether a quantitative increase in the complex abundance could modulate the stress response in the MT-ATP6 m.9205delTA fibroblasts. Stable retroviral overexpression of the wild-type AFG3L2 CDNA in these fibroblasts suppressed both the OPA1 processing and mitochondrial ribosomal phenotypes (Fig 4C). Because the complex can act as an AAA unfoldase independently of its role in proteolysis (Arlt et al, 1996), we sought to distinguish which function was most important during heat shock. To this end, we used site-directed mutagenesis to insert an E575Q mutation in the HEXXH motif of the AFG3L2 protease domain, although the protein still retains a functional AAA domain. A quantitative increase in the AAA function of AFG3L2 was epistatic to the translation of the m.9205delTA mutation during heat shock, suppressing both the OPA1 and the mitochondrial ribosomal phenotypes indistinguishably from the wild-type CDNA (Fig 4C). In contrast, AFG3L2 overexpression had no effect on the rapid attenuation of mitochondrial protein synthesis during heat shock (Fig 4D). Taken together, our findings revealed proteotoxicity arising from the translation of an aberrant mitochondrial mRNA and AFG3L2 dysfunction. The ability of the AFG3L2 complex to unfold these nascent chain substrates appears to be a critical modulator of this proteotoxicity arising from mitochondrial gene expression.

\section{Remodelling of lamellar cristae due to inhibition of mitochondrial protein synthesis}

The OPA1 stress-induced proteolytic processing associated with AFG3L2 dysfunction has been linked to alterations in cristae morphology (Merkwirth et al, 2008; Ehses et al, 2009; MacVicar \& Langer, 2016). However, our data clearly demonstrated this mitochondrial quality control step is also integrated with mitochondrial gene expression. Because the respiratory chain complexes and the $\mathrm{F}_{1} \mathrm{~F}_{\mathrm{O}}$ ATP synthase are localized within cristae (Rabl et al, 2009), biogenesis of these membrane invaginations is predicted to be coupled to the synthesis and assembly of the oxidative phosphorylation complexes. Therefore, we sought to distinguish primary versus potential feedback responses in cristae morphogenesis among these various factors. The ability to block OPA1 stress-activated

Figure 3. Heat shock during translation of an aberrant mitochondrial mRNA activates OMA1 and a ribosome decay pathway.

(A) Immunoblotting of whole-cell lysates from the MT-ATP6 m.9205delTA fibroblasts. (B) Left, immunoblotting of whole-cell lysates from the MT-ATP6 m.9205delTA fibroblasts. Right, quantification of the immunoblotting from three independent experiments. Data represent mean \pm SD. (C) Left, representative images of Northern blotting of total cellular RNA from MT-ATP6 m.9205delTA fibroblasts. Right, quantification of the Northern blotting from four independent experiments. Data represent mean \pm SD. (D) A representative image of Southern blotting for mitochondrial DNA copy number from the MT-ATP6 m.9205delTA fibroblasts from multiple independent experiments. (E) Quantitative label-free liquid chromatography-mass spectrometry/mass spectrometry analysis of mitochondrial ribosomal subunits from the large (LSU) and small (SSU) subunit of wild-type and MT-ATP6 m.9205delTA fibroblasts grown at $37^{\circ} \mathrm{C}$ or heat shocked for $4 \mathrm{~h}$ at $45^{\circ} \mathrm{C}$. Each data point represents a single mitochondrial ribosomal protein. Data were collected from isolated mitochondria from five independent experiments for each genotype and temperature condition. (F) Immunoblotting of whole-cell lysates of human fibroblasts with the indicated MT-ATP6 genotypes following culture at $37^{\circ} \mathrm{C}$ or heat shocked for the indicated time at $45^{\circ} \mathrm{C}$ treated with the indicated translation inhibitors. Anisomycin inhibits translation elongation on cytoplasmic ribosomes. 
A

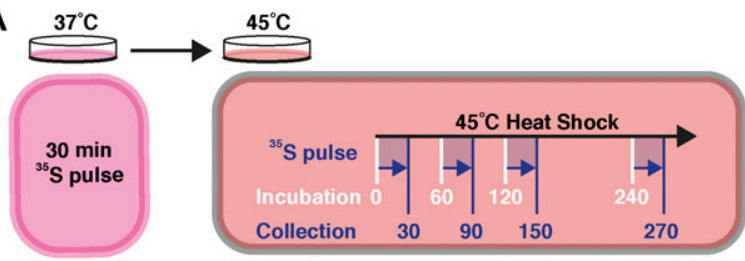

B
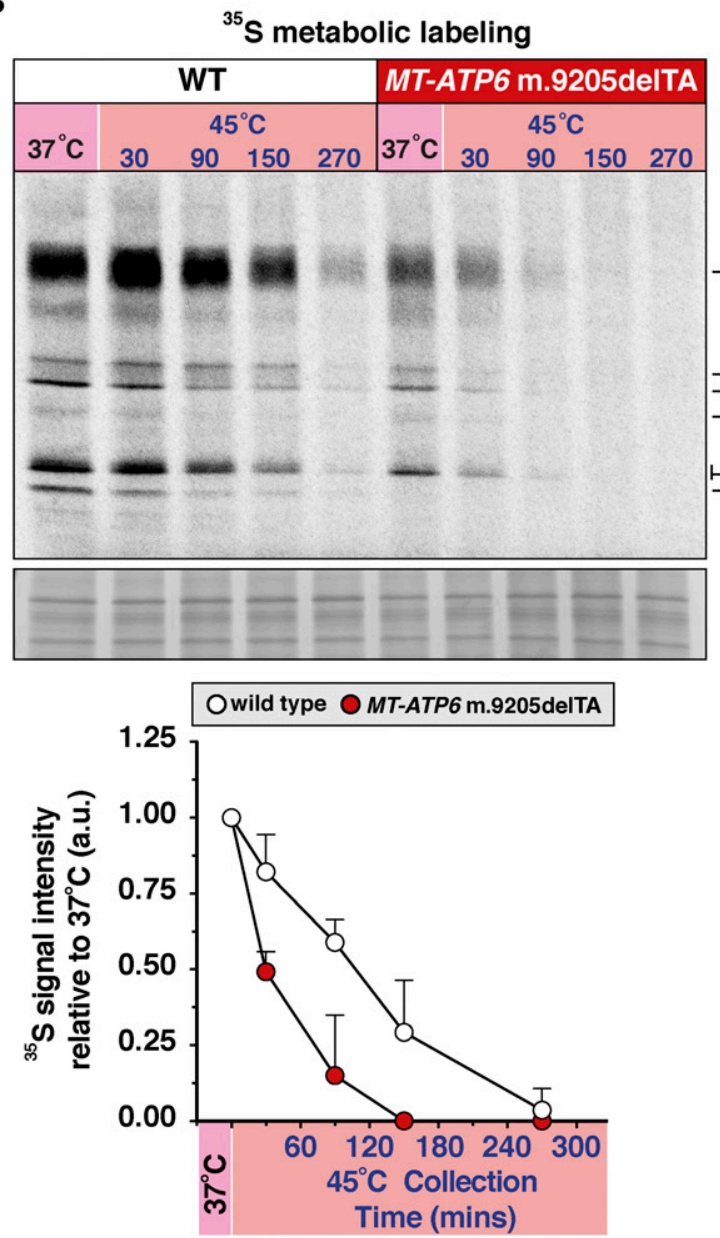

C

collection

time (mins)

MT-CO1

MT-CYTB

MT-ND2

MT-ND1

MT-CO2/3

MT-ATP6

coomassie

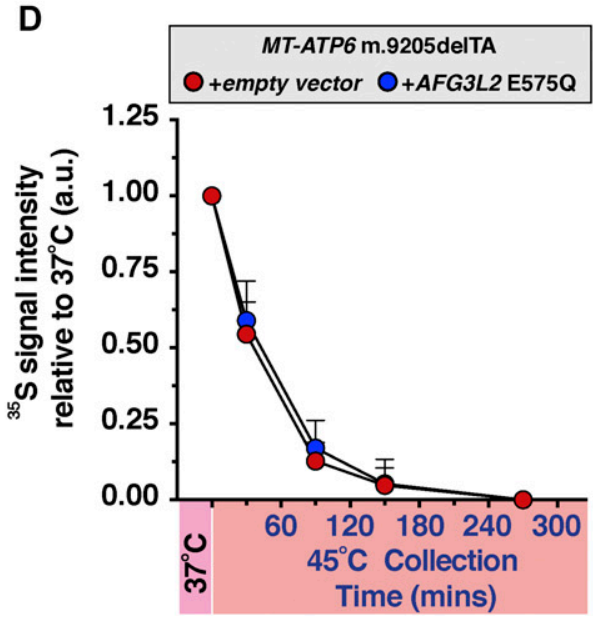

Figure 4. Translation of an aberrant mitochondrial mRNA negatively regulates protein synthesis during heat shock.

(A) A schematic illustrating the experimental workflow for ${ }^{35} \mathrm{~S}$-methionine/cysteine metabolic labelling for mitochondrial protein synthesis during heat shock. All cells were pulse labelled with ${ }^{35} \mathrm{~S}$-methionine/cysteine for $30 \mathrm{~min}$ to measure mitochondrial protein synthesis following the indicated incubation times at $45^{\circ} \mathrm{C}$. (B) Top, a representative metabolic labelling with ${ }^{35} \mathrm{~S}$-methionine/cysteine during heat shock in human fibroblasts with the indicated MT-ATP6 genotypes. Below, quantification of ${ }^{35} \mathrm{~S}$-incorporation into mitochondrial proteins during heat shock. Data represent mean + SD from four independent experiments. a.u., arbitrary units. (C) Human fibroblasts with the MT-ATP6 m.9205delTA genotype were stably transduced by retrovirus with an empty vector, wild-type AFG3L2 or E575Q AFG3L2 cDNA. Immunoblotting of whole-cell lysates from cells heat shocked for $4 \mathrm{~h}$ at $45^{\circ} \mathrm{C}$. ${ }^{*}$ Nonspecific band detected with the AFG3L2 antibody. (D) Quantification of ${ }^{35} \mathrm{~S}$-incorporation into mitochondrial proteins in human fibroblasts with the MT-ATP6 m.9205delTA genotype (from C). Data represent mean \pm SD from four independent experiments. All data are representative of multiple independent experiments.

processing with chloramphenicol (Figs 1, 2, 3, and 4) provided a robust tool to investigate the question.

In human fibroblasts, mitochondrial cristae appear as lamellar sheets with single or multiple crista junctions, either circular or slot-like in shape, when imaged in situ with electron tomography and 3D modelling (Fig $5 \mathrm{~A}-\mathrm{C}$, Video 1). Dimerization of the $\mathrm{F}_{1} \mathrm{~F}_{\mathrm{O}}$ ATP synthase generates positive curvature along cristae (Rabl et al, 2009; Davies et al, 2012). However, only with a severe assembly defect in the complex (e.g.,
ATP5B siRNA) did we observe a robust alteration in cristae morphology in transmission electron micrographs (Figs S2A and B, S3, and S4A), which appears similar to that seen with dimerisation defects (Rabl et al, 2009; Harner et al, 2016). In contrast, milder assembly defects or pathogenic MT-ATP6 mutations associated with human disease had lamellar cristae (Figs 5A-C, S2A and B, S3, S4B-D, and Video 1 and 2). Apparently, a low level of a functional $F_{1} F_{0}$ ATP synthase (Fig S3) is sufficient to maintain cristae curvature (Figs 5A and C, S4, and Video 1 and 2). 
䖪路 Life Science Alliance

A

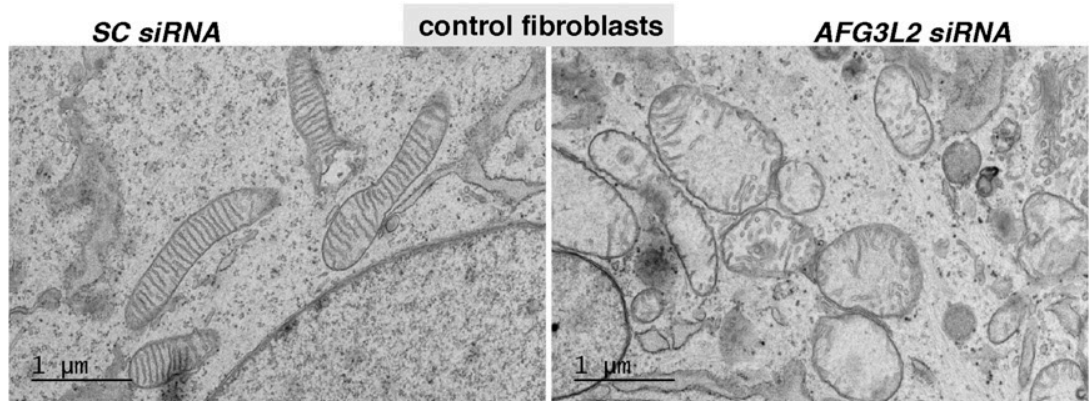

SC SiRNA

MT-ATP6 m.9205delTA

AFG3L2 SIRNA
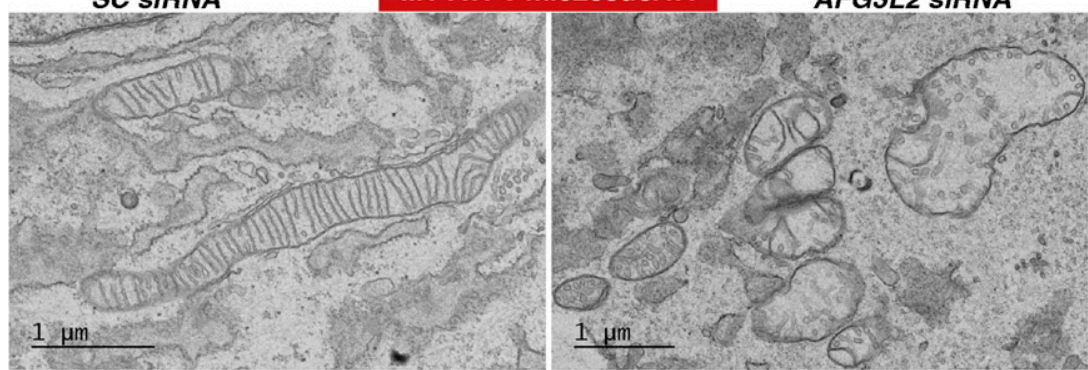

B

control fibroblasts

SC SiRNA

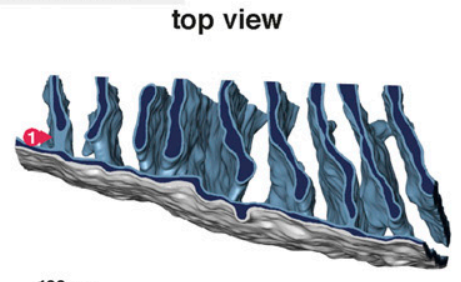

$\underline{100 \mathrm{~nm}}$

\section{AFG3L2 SIRNA}

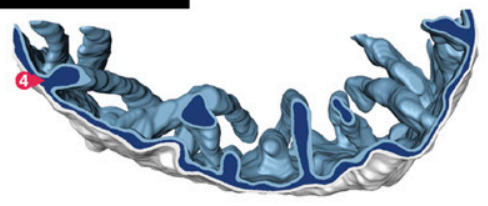

$\underline{100 \mathrm{~nm}}$

C

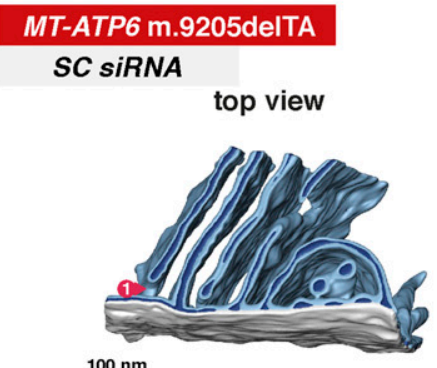

$100 \mathrm{~nm}$

\section{AFG3L2 SIRNA}

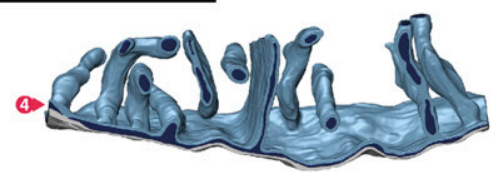

$\underline{100 \mathrm{~nm}}$

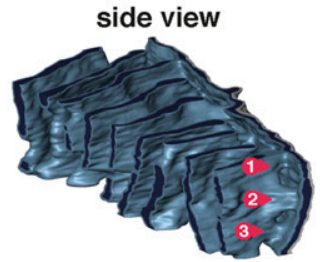

$\underline{100 \mathrm{~nm}}$

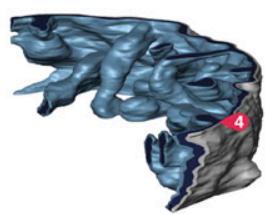

$\underline{100 \mathrm{~nm}}$

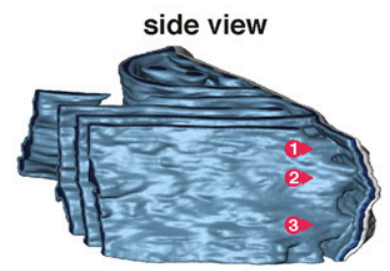

$100 \mathrm{~nm}$

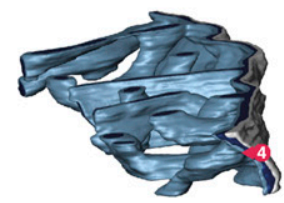

$100 \mathrm{~nm}$
IMS view

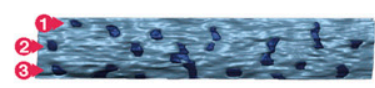

$100 \mathrm{~nm}$

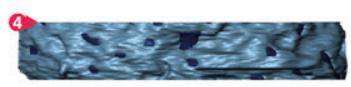

$100 \mathrm{~nm}$

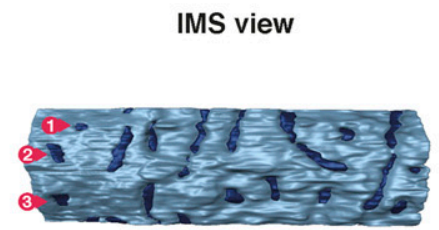

$100 \mathrm{~nm}$

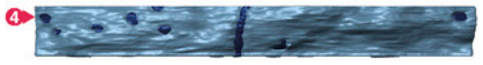

$100 \mathrm{~nm}$ 
In contrast, AFG3L2 siRNA induced mitochondrial swelling and disrupted the ordered lamellar ultrastructure: sheets collapsed, appearing as swollen tubes (Fig 5A-C and Video 3 and 4). Of note, tubular cristae still had intact circular junctions indistinguishable from those of wild-type (Fig $5 \mathrm{~B}$ and C) even with a robust loss of the long membrane-tethered OPA1 forms (Fig 1B). Chloramphenicol blocks the stress-induced OPA1 processing and mitochondrial fragmentation associated with AFG3L2 dysfunction (Fig 1B and D), but yet, we still observed remodelling of the inner membrane into short stubby cristae tubules and rings (Fig 6A). In fact, the inhibition of mitochondrial protein synthesis with chloramphenicol alone in wild-type fibroblasts dramatically remodelled the inner membrane without organelle fragmentation (Figs 1D and 6A). Short stubby cristae tubules were not observed with specific defects to the assembly of the $F_{1} F_{0}$ ATP synthase (Fig S4). Thus, a robust alteration in cristae morphogenesis appeared to arise with a global reduction in mitochondrial protein synthesis independent of OPA1 processing. This remodelling event could possibly serve as a feedback response to membrane biogenesis from defective assembly of all four oxidative phosphorylation complexes (I, III, IV, and V).

To further test this hypothesis, we examined the mitochondrial ultrastructure in two established cases with severe combined oxidative phosphorylation deficiencies but lacking stress-induced OPA1 processing: the mtDNA m.8344A $>\mathrm{G}$ tRNA ${ }^{\text {Lys }}$ mutation and C12orf65 deficiency. In myoblasts homoplasmic for the m.8344A>G tRNA ${ }^{\text {Lys }}$ mutation, we observed short stubby cristae that appeared as tubules and not lamellar sheets compared with myoblasts with the same nuclear background but wild-type mtDNA (Fig 6B). Furthermore, in human fibroblasts with C12orf65 deficiency (Antonicka et al, 2010), we also observed a similar cristae phenotype that was restored to lamellar sheets following functional complementation with retroviral expression of the wild-type C12orf65 CDNA (Fig 6C).

Last, we addressed how heat shock to the MT-ATP6 m.9205delTA fibroblasts affected mitochondrial morphology and ultrastructure. A 4-h heat shock did not induce mitochondrial fragmentation or alterations in the inner membrane ultrastructure (Fig 6D and E) even with robust stress-induced OPA1 processing (Figs 2D, 3A and F, and 4C). This would indicate that additional signals to the actual proteolytic processing of OPA1 are required during heat shock to trigger mitochondrial fragmentation. Our findings indicate how mitochondrial protein synthesis per se is a major functional determinant for cristae morphogenesis. Thus, any organelle stress response that impinges globally upon mitochondrial gene expression will initiate remodelling of the inner membrane ultrastructure.

\section{Discussion}

Proteotoxicity has long been considered a key factor in the molecular pathogenesis of mitochondrial dysfunction in human disease, but the sources and origin of the offending toxic substrates have remained unclear. Here, we demonstrated how disruptions to mitochondrial nascent chain quality control act as a proteotoxic trigger, initiating a stress response that links inner membrane ultrastructure and ribosome homeostasis. These are key features observed at the cell biological level in human patients and animal models with pathogenic mutations in AFG3L2 or SPG7 (Casari et al, 1998; Nolden et al, 2005; Almajan et al, 2012). Our data also establish the hierarchy to these molecular events, arising from the progressive failure in the quality control of mitochondrial nascent chain synthesis. Our findings did not identify the specific type of mitochondrial nascent chain proteotoxicity that occurs with AFG3L2 dysfunction. However, we provided experimental evidence by which the translation of a specific class of aberrant mitochondrial mRNA also activates the same stress response pathway. Thus, the proteotoxic trigger with AFG3L2 dysfunction may arise during translation of aberrant mitochondrial mRNAs. Currently, we have little understanding on the frequency by which mistakes are generated during the nucleolytic processing of the mitochondrial polycistronic RNA messages. Presumably, these errors in gene expression are resolved quickly by RNA surveillance mechanisms (Keiler, 2015) so as not to activate an organelle stress response. To test this attractive hypothesis requires experimentation beyond the scope of the present study but promises to be an exciting avenue of future research.

Mitochondrial dysfunction generates a heterogeneous collection of clinical disorders that cannot be explained simply by a defect in oxidative phosphorylation (Suomalainen \& Battersby, 2018). Understanding the specific molecular defects and ensuing stress responses is likely key to the pathogenesis. To this end, it is perhaps not surprising that we found only a single class of additional pathogenic mutation (MT-ATP6 m.9205delTA) that activated the mitochondrial translation stress response, but only following the stress of heat shock. Acute febrile infections are a critical event in the natural history of mitochondrial disorders (Thorburn et al, 2003; Magner et al, 2015). It could be argued the heat stress used in our experiments is high, but it is important to point out that we used cultured human skin fibroblasts, not neurons, which would be exposed to such temperatures under physiological conditions across the globe. Interestingly, the chaperone function of the AAA domain in the AFG3L2 complex, and not the proteolytic capacity, was critical for resolving the stress response initiated by nascent chain synthesis of the MT-ATP6 m.9205delTA during heat shock. Other mitochondrial AAA protease complexes, such as CLPXP, also have a distinct chaperone role independently of proteolytic function (Kardon et al, 2015). Collectively, the findings of this study demonstrated how modulating the rate of mitochondrial protein synthesis in a context-specific case can be beneficial to organelle homeostasis and cell fitness. Such an approach may be an avenue to develop for patients with AFG3L2 and SPG7 mutations.

Numerous studies have established OPA1 as a central factor for the dynamics and ultrastructure of mitochondrial membranes with

Figure 5. AFG3L2 dysfunction remodels mitochondrial inner membrane ultrastructure.

(A) Representative transmission electron micrographs of human fibroblasts with the indicated MT-ATP6 genotypes treated with siRNAs. SC, scrambled sequence. (B) Mitochondrial inner membrane morphology by electron tomographic models from three perspectives in wild-type control human fibroblasts treated with the indicated siRNAs. Numbers indicate the position of cristae junctions along a single crista invagination in different orientations. (C) Same as (B) but in human fibroblasts with the indicated MT-ATP6 genotype. IMS, intermembrane space. 
踽 Life Science Alliance

A
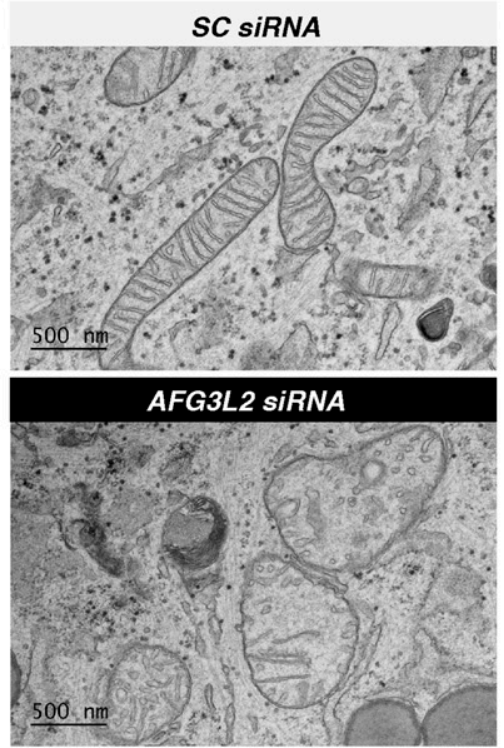

B
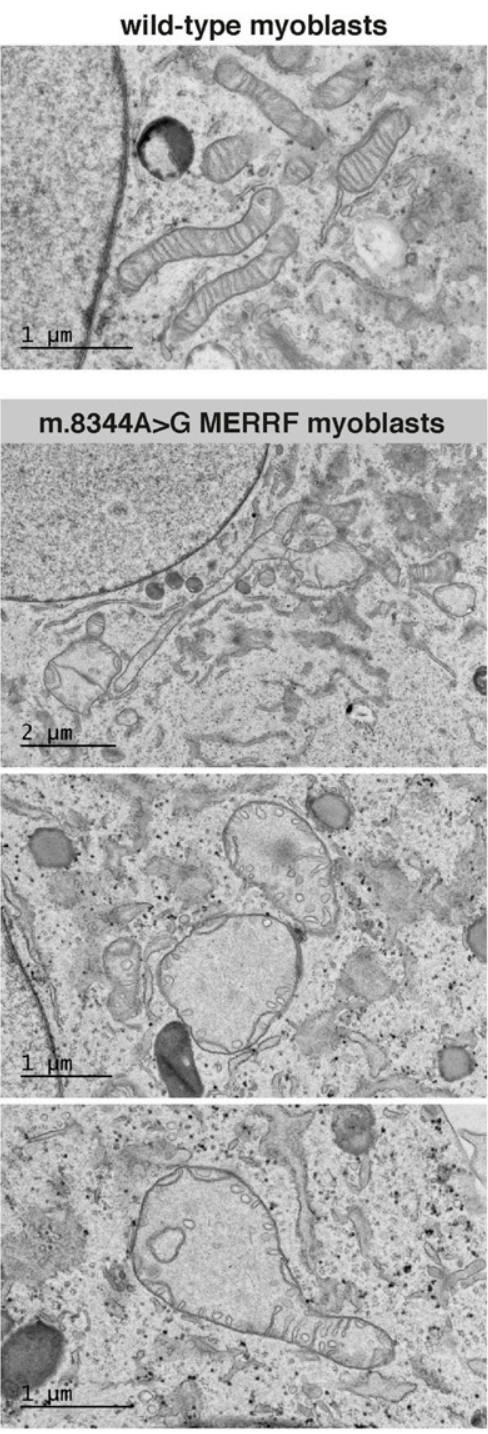

C
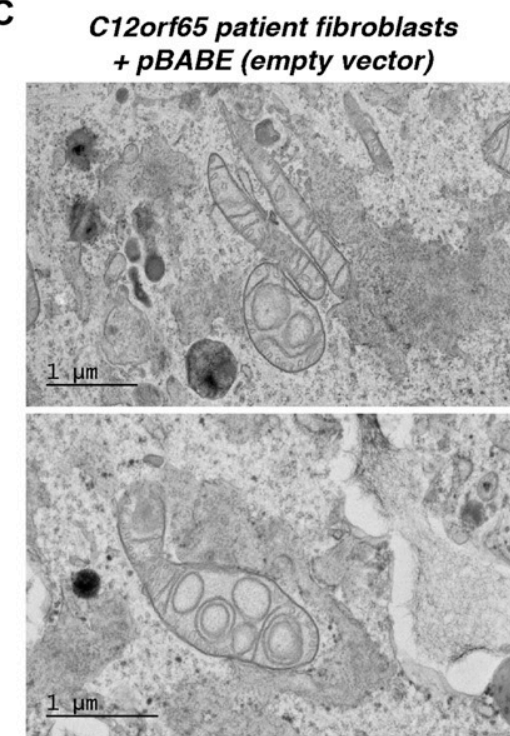

D

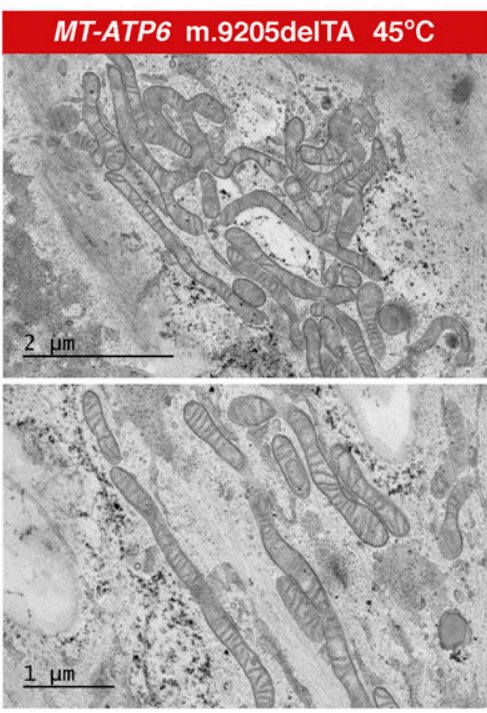

$S C$ siRNA + chloramphenico

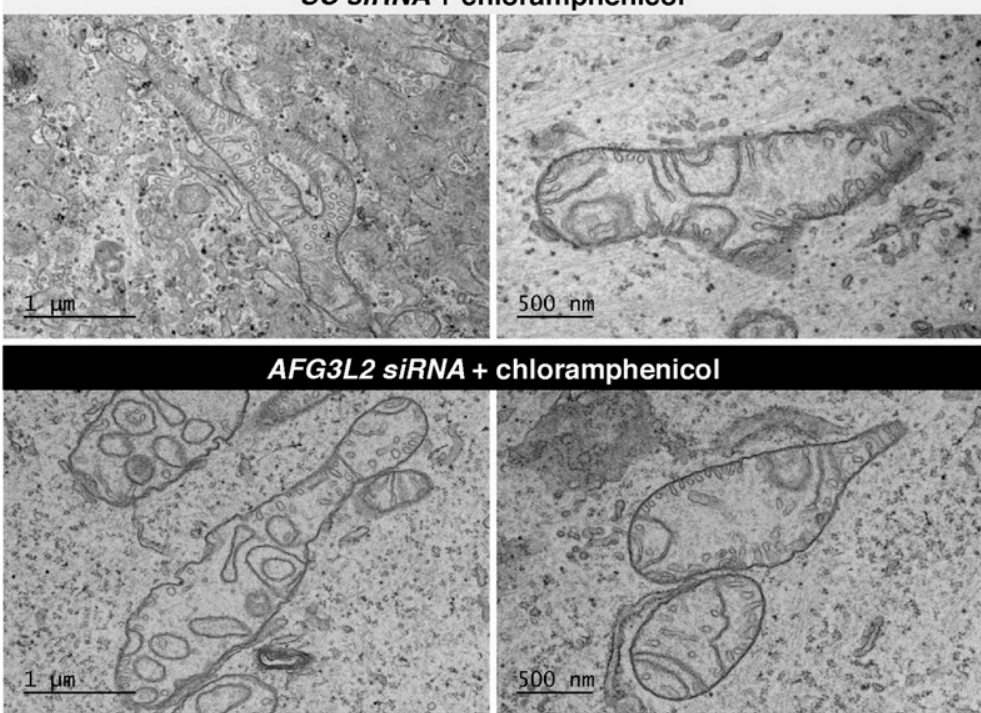

C12orf65 patient fibroblasts + wild-type C12orf65 cDNA

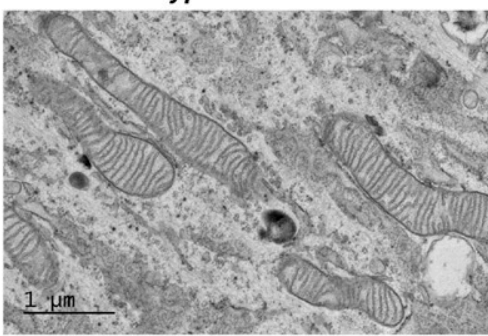

Human fibroblasts

$\Delta$ L84X-C12orf65

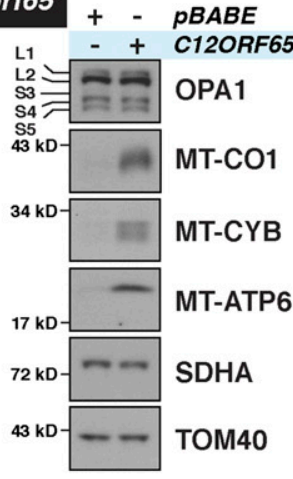

E

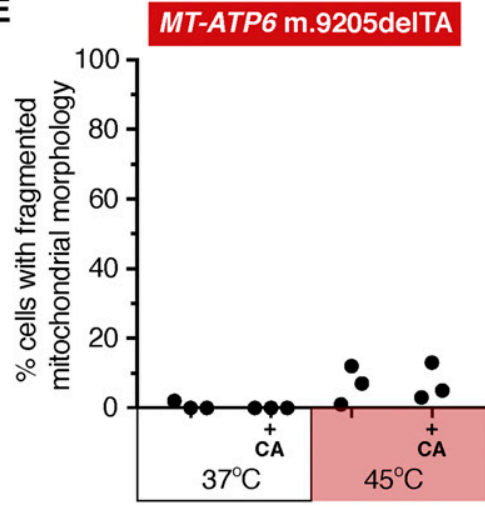


the proteolytic processing of the membrane-tethered form proposed as a key regulator step (Friedman \& Nunnari, 2014; MacVicar \& Langer, 2016). An outstanding question, however, remains on what is a direct function of OPA1 in cristae morphogenesis from an indirect or downstream response. Because OPA1 proteolytic processing is associated with pleiotropic phenotypes, it is important to resolve this distinction. Our data revealed dynamic cross-talk between OPA1 and mitochondrial protein synthesis, suggesting that many functions attributed to the stress-induced proteolytic processing of OPA1 could also be due to altered protein synthesis by mitochondrial ribosomes. Moreover, our electron tomograms of mitochondria in situ demonstrated OPA1 proteolytic processing is not accompanied with alterations to the cristae junctions or acute changes to the inner membrane ultrastructure. This is in contrast to observations from experiments with isolated mitochondria using the proapoptotic inducer BID (Frezza et al, 2006). Therefore, the long membrane-tethered OPA1 does not appear to play a strict role in cristae junction formation in all cases, suggesting the general applicability of this model should be reconsidered and may only be relevant to dying cells or isolated mitochondria.

Currently, the proximity of OPA1 and OMA1 to the sites of mitochondrial protein synthesis is not known, but the apparent tight coupling between these systems suggests a physical juxtaposition. Severing the OPA1 membrane tether appeared to correlate with a loss of mitochondrial gene expression in many cases. However, when the AFG3L2 E575Q mutant was overexpressed in the MT-ATP6 m.9205delTA background, we blocked OMA1 activation and ribosome decay but not the attenuation in mitochondrial protein synthesis associated with heat shock, suggesting that the OPA1 stress-induced proteolytic processing is not a regulatory switch for mitochondrial gene expression. Thus, the physiological rationale for this regulatory event remains unknown. The bulk of the investigations into the regulation of OMA1 activation have used ectopic addition of agents such as CCCP (Ishihara et al, 2006; Ehses et al, 2009; Head et al, 2009; Baker et al, 2014); however, these compounds are severely toxic to cells, producing a cellular state of a magnitude neither physiological nor relevant to human disease. The use of these compounds will not reveal the endogenous signals that trigger this key regulatory event in mitochondrial homeostasis. Future work should seek to determine the functional interplay between OPA1 and mitochondrial gene expression because our data robustly demonstrated failures in the quality control of nascent chain synthesis is a major OMA1 trigger.

Activation of the mitochondrial translation stress response induced mitochondrial ribosome decay. Previously, we showed how protein over-accumulation and dissipation of the membrane potential also induced mitochondrial rRNA and mRNA decay (Richter et $\mathrm{al}, 2013,2015)$ but occurred on a slower time scale than we observed in the MT-ATP6 m.9205delTA patient fibroblasts with heat shock. Mammalian mitochondrial ribosomes are composed of more than 80 individual proteins, two rRNAs, and a tRNA (Amunts et al, 2015; Greber et al, 2015) and require a plethora of factors to coordinate assembly many of which are now being discovered (Ott et al, 2016; Brown et al, 2017). Our data provide an example of organelle dysfunction that triggers a degradation process specific to mitochondrial ribosomes and show the relevance of this decay as a molecular mechanism in the pathogenesis of human disease. These matrix localized degradation pathway(s) will be independent of processes that involve large-scale organelle turnover, such as mitophagy, because we did not observe any changes to mitochondrial DNA copy number or core import machinery (e.g., TOM40) that are degraded in Parkin-mediated mitophagy (Yoshii et al, 2011). In the cytosol, ribosome degradation is mediated by RNA surveillance pathways following severe aberrations in translation elongation (Lykke-Andersen \& Bennett, 2014). The mechanisms for these processes in mitochondria are largely unknown but open up an exciting avenue for future research.

\section{Materials and Methods}

\section{Cell culture}

Human and mouse fibroblasts, and cell lines were cultured at $37^{\circ} \mathrm{C}$ and $5 \% \mathrm{CO}_{2}$ in Dulbecco's modified Eagle's medium (Lonza) with high glucose supplemented with $10 \%$ fetal bovine serum, $1 \times$ glutamax, and $50 \mu \mathrm{g} / \mathrm{ml}$ uridine. All primary cells were immortalized by expressing E7 and hTERT (Lochmüller et al, 1999) unless previously immortalized by the donating laboratory. All cells tested negative for mycoplasma infection (PromoKine). Human myoblast cultures (kind gift from Eric Shoubridge) were grown in myoblast medium from Lonza. Chloramphenicol (Sigma-Aldrich) was used at a dose of $400 \mu \mathrm{g} / \mathrm{ml}$. Heat shock was administered by adding pre-warmed media to the plate and immediate transfer to an incubator set at $45^{\circ} \mathrm{C}$. In the case of chloramphenicol treatment, the cells were treated for $72 \mathrm{~h}$ before and during heat shock. Stealth siRNAs (Thermo Fisher Scientific: AFG3L2, HSS116886, 5'-GAGUAGUAGACAGAUUGGAAGUCGU; Atp5b, MSS202262, 5'-GAUCACCUCGGUGCAGGCUAUCUAU; PHB2-1, HSS117606, 5' -ACGAUCGCCACAUCACAGAAUCGUA; PHB2-2, HSS11760 4, 5' - CCAAAGACCUACAGAUGGUGAAUAU; STOML2, HSS121274, 5' -GCCUCCGUUAUGAGAUCAAGGAUAU; TMEM70, HSS123635, 5'-CGAGUCUGAUUGGCCUUACAUUUCU; and scrambled control, 12935300) were transfected on day 1 and 3 with Lipofectamine RNAiMAX (Thermo Fisher Scientific) and collected on day 8 for analysis unless indicated otherwise. All siRNA knockdowns were evaluated by immunoblotting using specific antibodies. Retrovirus was generated by transient transfection of retroviral plasmids into

\section{Figure 6. Global defects to mitochondrial protein synthesis remodels cristae ultrastructure.}

(A) Representative transmission electron micrographs of human fibroblasts treated with the indicated siRNAs with or without chloramphenicol (CA). (B) Representative transmission electron micrographs of human myoblasts with the same nuclear genetic background that are either homoplasmic for wild-type mitochondrial DNA (mtDNA) or the m.8344A>G tRNA ${ }^{\text {Lys }}$ mutation. (C) Representative transmission electron micrographs of human fibroblasts from a patient with a pathogenic mutation in C12orf65 stably transduced with an empty vector or the wild-type C12orf65 cDNA. Bottom right, immunoblotting of whole-cell lysates with the indicated antibodies. (D) Representative transmission electron micrographs of human fibroblasts with the MT-ATP6 m.9205delTA mutation heat shocked for $4 \mathrm{~h}$ at $45^{\circ} \mathrm{C}$. (E) Scatter plot quantification of mitochondrial morphology under light microscopy in human fibroblasts with the MT-ATP6 m.9205delTA mutation grown at $37^{\circ} \mathrm{C}$ or heat shocked for $4 \mathrm{~h}$ at $45^{\circ} \mathrm{C}$ with or without CA. Data are from three independent experiments. SC, scrambled sequence. 
the Phoenix amphotropic packaging line. The cells were used directly in experiments following selection with puromycin or blasticidin.

\section{Cloning}

Wild-type cDNAs (AFG3L2, clone BC065016; COX10, clone BC000060) were obtained from the ORFeome or Mammalian Genome Collection. These cDNAs were in a Gateway entry vector (pENTR221 or pCMV-SPORT6) or cloned into pDONR201 using PCR with KAPA HiFi followed by recombination. All full-length cDNAs were recombined into Gateway-converted pBABE-puro and pMXs-IRES-Blasticidin with LR Clonase II (Thermo Fisher Scientific). All cDNAs were thoroughly sequenced by Sanger sequencing for verification initially and after all PCR manipulations. Site-directed mutagenesis of E575Q in AFG3L2 was performed using Agilent primer design with PCR (KAPA HiFi), purification, Dpnl (NEB) digest, and transformation into XL1 Blue chemically competent cells. Sanger sequencing was used to validate mutagenesis. Mutant cDNA was recombined into Gateway-converted pBABE-puro and pMXs-IRES-Blasticidin with LR Clonase II.

\section{Immunoblotting}

Cells were solubilized in phosphate-buffered saline, 1\% dodecylmaltoside (DDM), $1 \mathrm{mM}$ PMSF, and complete protease inhibitor (Thermo Fisher Scientific). Protein concentrations were measured by the Bradford assay (Bio-Rad). Equal amounts of proteins were separated by Tris-glycine SDS-PAGE and transferred to nitrocellulose by semi-dry transfer or by Tricine-SDS-PAGE onto polyvinylidene difluoride for proteins smaller than 20 kD (Schägger, 2006). Membranes were blocked in Tris-buffered saline, $0.1 \%$ Tween 20 (TBST) with $1 \%$ milk at room temperature for $1 \mathrm{~h}$ and then primary antibodies were incubated overnight at $+4^{\circ} \mathrm{C}$ in $5 \%$ BSA/TBST and detected the following day with secondary HRP conjugates (Jackson ImmunoResearch) using ECL with film. Primary antibodies from Proteintech Group: AFG3L2 (14631-1-AP, 1:5,000); ATP5B (172471-AP, 1:5,000); uL11m (15543-1-AP, 1:20,000); bS18b (16139-1-AP, 1: 2,000); mS26 (15989-1-AP, 1:5,000); mS27 (17280-1-AP, 1:5,000); MTATP6 (55313-1-AP, 1:2,000); MT-CYB (55090-1-AP, 1:1,000); PHB2 (66424-1-Ig, 1:5,000); STOML2 (10348-1-AP, 1:5,000); and TMEM70 (20388-1-AP, 1:5,000). Primary antibodies from Abcam/Mitosciences: MT-CO1 (1D6E1A8, 1:500) and SDHA (C2061/ab14715, 1:10,000), and primary antibody from Santa Cruz: TOM40 (sc-11414, 1:5,000). Representative data of independent experiments were cropped in Adobe Photoshop with only linear corrections to brightness applied.

\section{TMRM staining}

HEK293 cells were treated with siRNAs against AFG3L2 and scrambled sequence then stained with 200 nM TMRM (Life Technologies). For a positive control, the cells were treated with CCCP $(10 \mu \mathrm{M})$. After TMRM staining, the cells were analyzed by flow cytometry (Accuri C6, Becton Dickinson or a BD LSR II). Independent experiments were performed and quantified. All siRNA experiments were subsequently validated by immunoblotting.

\section{Metabolic labelling of mitochondrial protein synthesis}

Mitochondrial protein synthesis was analyzed by metabolic labelling with ${ }^{35} \mathrm{~S}$ methionine/cysteine (Richter et al, 2015). The cells were pretreated with anisomycin $(100 \mu \mathrm{g} / \mathrm{ml})$ to inhibit cytoplasmic translation then pulsed with $200 \mu \mathrm{Ci} / \mathrm{ml}{ }^{35} \mathrm{~S}$ Met-Cys (EasyTagPerkin Elmer). In chase experiments, the cells were pulsed for 30 min with radiolabel then the medium removed and replaced with fresh medium lacking the radioisotope for the indicated time. Equal amounts of sample protein were first treated with Benzonase (Thermo Fisher Scientific) on ice and then resuspended in $1 \times$ translation loading buffer (186 mM Tris-Cl, pH 6.7, 15\% glycerol, 2\% SDS, $0.5 \mathrm{mg} / \mathrm{ml}$ bromophenol blue, and $6 \% \beta$-mercaptoethanol). 12\%-20\% gradient Tris-glycine SDS-PAGE gels were used to separate the samples and then dried for exposure with a phosphoscreen and scanned with a Typhoon 9400 or Typhoon FLA 7000 (GE Healthcare) for quantification. The gels were rehydrated in water and Coomassie stained to confirm loading.

\section{Mass spectrometry analysis}

Mitochondria were isolated (QIAGEN) from control and m.9205delTA MT-ATP6 fibroblasts cultured at $37^{\circ} \mathrm{C}$ or following a 4 -h heat shock at $45^{\circ} \mathrm{C}$. Mitochondria were lysed (PBS, $1 \%$ DDM, $1 \mathrm{mM}$ PMSF, and complete protease inhibitor [Thermo Fisher Scientific]) on ice for $20 \mathrm{~min}$ and then centrifuged at 20,000 $\mathrm{g}$ for $20 \mathrm{~min}$ at $+4^{\circ} \mathrm{C}$. Insoluble materials were discarded and proteins in the supernatant were precipitated with acetone.

The protein pellet was dissolved in ProteaseMax (Promega) in $50 \mathrm{mM} \mathrm{NH}_{4} \mathrm{HCO}_{3}$, and the proteins were reduced, alkylated, and insolution digested with trypsin (Promega) according to the manufacturer's instructions. Peptides were desalted and concentrated before mass spectrometry by the STAGE-TIP method using a C18 resin disk (3M Empore). The peptides were eluted twice with $0.1 \%$ formic acid $/ 50 \%$ ACN, dried, and solubilized in $7 \mu \mathrm{l} 0.1 \%$ formic acid for mass spectrometry analysis. Each peptide mixture was analyzed on an Easy nLC1000 nano-LC system connected to a quadrupole Orbitrap mass spectrometer (QExactivePlus; Thermo Electron) equipped with a nanoelectrospray ion source (EasySpray/Thermo Fisher Scientific). For the liquid chromatography separation of the peptides, we used an EasySpray column capillary of $25 \mathrm{~cm}$ bed length (C18, $2 \mu \mathrm{m}$ beads, $100 \AA, 75 \mu \mathrm{m}$ inner diameter, Thermo). The flow rate was $300 \mathrm{nl} / \mathrm{min}$, and the peptides were eluted with a $2 \%-30 \%$ gradient of solvent B in 60 min. Solvent A was aqueous $0.1 \%$ formic acid and solvent B 100\% acetonitrile/0.1\% formic acid. The data-dependent acquisition automatically switched between MS and MS/MS mode. Survey full scan MS spectra were acquired from a mass-to-charge ratio $(\mathrm{m} / \mathrm{z})$ of 400 to 1,200 with the resolution $\mathrm{R}=$ 70,000 at $\mathrm{m} / \mathrm{z} 200$ after accumulation to a target of 3,000,000 ions in the quadruple. For MS/MS, the 10 most abundant multiple-charged ions were selected for fragmentation on the high-energy collision dissociation cell at a target value of 100,000 charges or maximum acquisition time of $100 \mathrm{~ms}$. The MS/MS scans were collected at a resolution of 17,500. Target ions already selected for MS/MS were dynamically excluded for $30 \mathrm{~s}$.

The resulting MS raw files were submitted to the MaxQuant software version 1.6.1.0 for protein identification using the 
Andromeda search engine. The UniProt human database (October 2017) was used for database searches. Carbamidomethyl (C) was set as a fixed modification, and protein $\mathrm{N}$-acetylation and methionine oxidation were set as variable modifications. First search peptide tolerance of $20 \mathrm{ppm}$ and main search error $4.5 \mathrm{ppm}$ were used. Trypsin without proline restriction enzyme option was used, with two allowed miscleavages. The minimal unique + razor peptides number was set to 1 , and the allowed false discovery rate was 0.01 (1\%) for peptide and protein identification. Label-free quantitation () was used with default settings. Known contaminants as provided by MaxQuant and identified in the samples were excluded from further analysis. Statistical analysis was performed with Perseus software version 1.5.6.0. The label-free quantitation data were log10 transformed, filtered to include only proteins, which were quantified in at least in three of five replicates in at least one group, and missing values were imputed with values representing a normal distribution with default settings in Perseus. To find statistically significant differences between the groups, $t$ test was performed using permutation-based false discovery rate with 0.05 cut off (Tyanova et al, 2016a, 2016b). The MS data are available in the PRIDE database under accession number PXD012416.

\section{Southern blotting}

Total DNA was isolated from cultured fibroblasts as described in QIAamp DNA Mini Kit (QIAGEN). DNA ( $5 \mu \mathrm{g})$ was digested with Pvull, then separated on a $0.8 \%$ agarose gel followed by alkaline transfer to Hybond-XL membrane (GE Healthcare). Two oligonucleotide probes for mtDNA (5'-GGCTCCAGGGTGGGAGTAGTTCCCTGC; 5' CCTCCCGAATCAACCCTGACCCCTCTCC) and three for $18 \mathrm{~S}$ rDNA (5'GGCCCGAGGTTATCTAGAGTCACC; 5'-TATTCCTAGCTGCGGTATCCAGGC; and 5'-ACCATCCAATCGGTAGTAGCGACG) were $5^{\prime}$ end labelled with $\gamma^{32} \mathrm{P}$ ATP by T4 polynucleotide kinase (NEB) and hybridized (50\% formamide, 7\% SDS, 0.25M sodium phosphate, pH 7.2, $10 \mathrm{mM}$ EDTA, $\mathrm{pH} 8.0,0.24 \mathrm{M} \mathrm{NaCl}$ ) overnight at $37^{\circ} \mathrm{C}$. Membranes were washed once with $2 \times$ SSC $/ 0.1 \%$ SDS for $60 \mathrm{~min}$, followed by $0.5 \times$ SSC $/ 0.1 \%$ SDS for $60 \mathrm{~min}$, and finally in $0.1 \times$ SSC $/ 0.1 \%$ SDS for $30 \mathrm{~min}$. All washings were performed at $37^{\circ} \mathrm{C}$. The membranes were dried and then exposed to a Phosphoscreen (GE Healthcare) and scanned with a Typhoon 9400 (GE Healthcare).

\section{Northern blotting}

Total cellular RNA was isolated using Trizol (Invitrogen) according to the manufacturer's instructions. For all samples, $5 \mu \mathrm{g}$ of total RNA was run through $1.2 \%$ agarose-formaldehyde gels and transferred to Hybond- $\mathrm{N}^{+}$membrane (GE Healthcare) by neutral transfer. T4 polynucleotide kinase (NEB) 5'-radiolabelled oligonucleotides were used for detection of mitochondrial transcripts. Oligonucleotides: 12S, 5'-GTTAATCGTGTGACCGCGGTGGCTGGC; 16S, 5'-GCTGTGTTATGCCCGCCTCTTCACGGG; and 18S, 5'-ACCATCCAATCGGTAGTAGCGACG. Hybridization (25\% formamide, 7\% SDS, 1\% BSA, $0.25 \mathrm{M}$ sodium phosphate, $\mathrm{pH} 7.2,1 \mathrm{mM}$ EDTA, pH 8.0, and $0.25 \mathrm{M}$ $\mathrm{NaCl}_{2}$ ) was performed for $16-20 \mathrm{~h}$ at $37^{\circ} \mathrm{C}$. Membranes were washed ( $2 \times$ SSC $/ 0.1 \%$ SDS) and then dried for exposure on a phosphoscreen (GE Healthcare) and scanned with a Typhoon 9400 (GE Healthcare).

\section{Amplicon generation and Illumina sequencing}

Genomic DNA was extracted from patient cells known to contain m.9205.delTA and wild-type control cells (Flp-In T-REx 293; Thermo Fisher Scientific) using NucleoSpin Tissue kit (Macherey-Nagel GmbH \& Co. KG) according to the manufacturer's instructions. Three PCR amplicons spanning the deletion site were designed (amplicon 1 [3.90 kb] [primers 5'-tgtaaaacgacggccagtCGCAAGTAGGTCTACAAGACG; 5'-caggaaacagctatgaccATAGAGAGGTAGAGTTTTTTCGTG]; amplicon 2 [3.16 kb] [primers 5'-tgtaaaacgacggccagtATTCTTATCCTACCAGGCTTCG; 5'-caggaaacagctatgaccAATGTTGAGCCGTAGATGCC]; and amplicon 3 [2.49 kb] [primers 5'-tgtaaaacgacggccagtCCCCATACTAGTTATTATCGAAACC; 5'-caggaaacagctatgaccGGCTTCGACATGGGCTTT]) and amplified in 20- $\mu$ l reactions with Phusion High-Fidelity DNA Polymerase (New England Biolabs). Cycling conditions were as follows: $95^{\circ} \mathrm{C}$ for $1 \mathrm{~min}, 35$ cycles of $95^{\circ} \mathrm{C}$ for $30 \mathrm{~s}, 60^{\circ} \mathrm{C}$ for $30 \mathrm{~s}$, and $72^{\circ} \mathrm{C}$ for $2 \mathrm{~min}$ followed by $72^{\circ} \mathrm{C}$ for $3 \mathrm{~min}$. The PCR products were purified using NucleoSpin Gel and PCR clean-up kit (MachereyNagel GmbH \& Co. KG) according to the manufacturer's instructions including second wash step and elution twice to $15 \mu \mathrm{l}$ of elution buffer. Library preparations and Illumina sequencing reactions were performed by the Max Planck-Genome-Centre Cologne, Germany (http://mpgc.mpipz.mpg.de/home/).

Sequencing reads were trimmed with Flexbar v2.5 (Dodt et al, 2012) for TruSeq adapters and base call quality (default parameters except -q 28 -m 50 -ae ANY -ao 10). Reads containing the M13 tag sequences in forward or reverse orientation were removed by Flexbar (parameters - bo 15 -bt 2 -bu -be ANY) to avoid primer-containing reads and highly biased coverage at the amplicon edges. Unassigned reads (without the M13 tag) were aligned with BWA v0.7.12-r1039 invoking mem (Li \& Durbin, 2010; Li, 2013) (parameters -T 19 -B 4 - L 5,4) to the human mitochondrial reference sequence (NC_012920.1). Uniquely aligned reads (SAMtools view - q 1) were converted to bam format, sorted, and indexed with SAMtools ( $\mathrm{Li}$ et al, 2009). Per base coverage was determined with bedtools v2.22.1 (Quinlan \& Hall, 2010) genomecov (parameters -split -d). Variants were detected with Lofreq* v2.1.2 (Wilm et al, 2012) as follows. First, the indel qualities were set by command lofreq indelqual -dindel, and then variants were called including indels with minimum base call quality 30 by command lofreq call-parallel -pp-threads 20 -N -B -q 30 -Q 30 -call-indels -no-default-filter. Variant results were further filtered for minimum variant quality Phred score 70 and maximum strand bias Phred score 60 (strand bias filter applied only if $\geq 85 \%$ of the variant supporting reads were on a single strand) by command lofreq filter -no-defaults -snvqualthresh 70 -sb-incl-indels -B 60. Next, the variants were filtered for minimum of 15 variant supporting reads using snpsift filter with the expression $D P^{\star} A F \geq 15$ and for minimum of three variant supporting reads on each strand (expression DP4[2] $\geq 3 \&$ DP4 $[3] \geq 3$ ). Finally, only variants and indels present at positions covered by minimum 100,000 reads and minimum variant allele frequency (AF value) of $1 \%$ were accepted. To understand the level of the reference genome bias, the aforementioned data analysis steps were repeated using a reference genome containing the deletion (9206.delA and 9205.delT). The detected indel frequencies were then compared. 


\section{Isokinetic sucrose gradients}

Medium from cultured cells grown on a 150-mm plate was rapidly removed, and the cells were flash-frozen on dry ice. The plates were immediately transferred to wet ice to thaw. The cells were washed two times with ice-cold PBS, lysed on the plate (50 mM Tris, $\mathrm{pH} 7.2$, $10 \mathrm{mM} \mathrm{Mg}(\mathrm{Ac})_{2}, 40 \mathrm{mM} \mathrm{NH}_{4} \mathrm{Cl} 100 \mathrm{mM} \mathrm{KCl}, 1 \%$ DDM, 1 mM ATP, $400 \mu \mathrm{g} /$ $\mathrm{ml}$ chloramphenicol, and $1 \mathrm{mM}$ PMSF), and transferred to a $1.5-\mathrm{ml}$ centrifuge tube for a 20-min incubation on ice. The samples were centrifuged for $10 \mathrm{~min}$ at $20,000 \mathrm{~g}$ at $4^{\circ} \mathrm{C}$. The supernatant was loaded on top of a $16-\mathrm{ml}$ linear $10 \%-30 \%$ sucrose gradient $(50 \mathrm{mM}$ Tris, $\mathrm{pH} 7.2,10 \mathrm{mM} \mathrm{Mg}(\mathrm{Ac})_{2}, 40 \mathrm{mM} \mathrm{NH}_{4} \mathrm{Cl} 100 \mathrm{mM} \mathrm{KCl}, 1 \mathrm{mM} \mathrm{ATP}$, $400 \mu \mathrm{g} / \mathrm{ml}$ chloramphenicol, and $1 \mathrm{mM}$ PMSF) and centrifuged for $15 \mathrm{~h}$ at $4^{\circ} \mathrm{C}$ and $74,400 \mathrm{~g}$ (Beckman SW $32.1 \mathrm{Ti}$ ). 24 equal-volume fractions were collected from the top for either trichloroacetic acid precipitation for protein immunoblotting or for RNA isolation.

\section{Light microscopy}

Fibroblasts were grown on coverslips and washed several times in PBS, fixed in 4\% paraformaldehyde for 15 min, washed in PBS, then treated with $100 \%$ methanol for 5 min, and washed again in PBS. The cells were blocked in 5\% BSA/PBST (PBS + 0.1\% Tween 20) and then incubated with rabbit anti-TOM20 (sc-11414, 1:250; Santa Cruz) for $1 \mathrm{~h}$ at room temperature or overnight $+4^{\circ} \mathrm{C}$. The cells were washed several times in PBST before incubation with anti-rabbit Alexa 4881 : 1,000 (Life Technologies) for $1 \mathrm{~h}$ and then washed several times in PBST. Coverslips were mounted with DABCO/MOWIOL on glass slides for imaging with a Zeiss inverted microscope at room temperature using a Plan-Apochromat 63×/1.40 Oil and slider assembly ApoTome2. Images were exported into ImageJ using the Fiji plug-in to apply brightness and contrast adjustments.

\section{Transmission electron microscopy}

Cultured cells were grown on glass coverslips and then fixed with

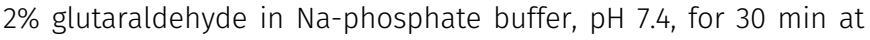
room temperature. After fixing, the samples were washed $3 \times 2$ min with Na-phosphate buffer, pH 7.4. The samples were then post-fixed with $1 \%$ osmium tetroxide for $1 \mathrm{~h}$ at room temperature, dehydrated with a graded series of ethanol (70\%, 96\%, and 100\%), incubated with transitional solvent acetone, and finally embedded gradually in Epon (TAAB). Images were acquired on a Jeol JEM-1400 transmission electron microscope with a Gatan Orius 1000B bottommounted CCD camera.

\section{Electron tomography and modelling}

Samples were prepared as described for TEM. Dual axis tilt series (Mastronarde, 1997) were recorded from one or consecutive semi-thick (250 nm) sections using Tecnai FEG 20 (FEI Company) microscope operating at $200 \mathrm{kV}$. The sections were tilted at onedegree intervals using a high-tilt specimen holder (model 2020; E.A. Fischione Instruments) between $\pm 62^{\circ}$. Images were acquired with SerialEM software (Mastronarde, 2005) using a $4 \mathrm{k} \times 4 \mathrm{k}$ Ultrascan 4000 CCD camera (Gatan Corporation) at nominal magnification of $9,600 \times$ or $11,500 \times$. The alignment of the tilt series and reconstructions were done with IMOD software package (Kremer et al, 1996) using 10-nm colloidal gold particles as fiducial markers. Tomographic reconstructions were segmented with MIB software (Belevich et al, 2016) and visualization was performed using Amira (VSG, FEI Company).

\section{High-resolution respirometry}

Oxygen consumption rates were determined by substrateuncoupler-inhibitor titration protocols in permeabilized human fibroblasts treated with siRNA using a high-resolution oxygraph (OROBOROS instrument). Values of specific oxygen consumption rates determined as $\mathrm{pmol} /\left(\mathrm{s}^{\star}\right.$ millions of cells) and are given as ratios of different respiration states. Briefly, $\sim 2 \times 10^{6}$ cells were resuspended in MiR05 (0.5 mM EGTA, $3 \mathrm{mM} \mathrm{MgCl}_{2}, 60 \mathrm{mM}$ lactobionic

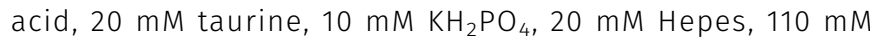
D-sucrose, and $1 \%$ fat-free BSA) and used per measurement. Oxygen consumption rates were measured in digitonin-permeabilized ( $1 \mu \mathrm{l}$ of $8 \mathrm{mM}$ per $1 \times 10^{6}$ cells) in the presence of pyruvateglutamate-malate (state 4 respiration on complex I), after addition of ADP (state 3 respiration on complex I), succinate (state 3 respiration on complex I and II), FCCP titration (maximal uncoupled respiration), and finally ascorbate with TMPD followed by azide (complex IV activity).

\section{Supplementary Information}

Supplementary Information is available at https://doi.org/10.26508/lsa. 201800219.

\section{Acknowledgements}

We thank LS Churchman; E Dufour, C Dunn, K Holmström, H Jacobs, T Langer, $\checkmark$ Paavilainen, and E Rugarli for valuable discussion; EA Shoubridge, T Langer, and $\mathrm{CL}$ Otin for generously sharing reagents; and the Max Planck-GenomeCentre Cologne, the University of Helsinki Electron Microscopy Unit and Genome Biology Unit. This work was supported by the Academy of Finland Centre of Excellence on Mitochondria to BJ Battersby; an Academy of Finland postdoctoral award (to U Richter); the Ella and Georg Ehrnrooth Foundation (to F Suomi). RW Taylor is supported by the Wellcome Centre for Mitochondrial Research (203105/Z/16/Z), the Medical Research Council Centre for Translational Research in Neuromuscular Disease, Mitochondrial Disease Patient Cohort (UK) (G0800674), the Lily Foundation, and the UK NHS Highly Specialised Service for Rare Mitochondrial Disorders of Adults and Children.

\section{Author Contributions}

U Richter: conceptualization, formal analysis, and investigation. KY Ng: conceptualization, formal analysis, and investigation.

F Suomi: conceptualization, formal analysis, and investigation.

P Marttinen: formal analysis and investigation.

T Turunen: formal analysis and investigation.

C Jackson: formal analysis and investigation.

A Suomalainen-Wartiovaara: resources.

$\mathrm{H}$ Vihinen: formal analysis and methodology.

E Jokitalo: formal analysis and methodology. 
TA Nyman: formal analysis and investigation. MA Isokallio: formal analysis and investigation.

JB Stewart: formal analysis and investigation.

C Mancini: resources.

A Brusco: resources.

S Seneca: resources.

A Lombès: resources.

RW Taylor: resources.

BJ Battersby: conceptualization, formal analysis, supervision, funding acquisition, investigation, project administration, and writingoriginal draft, review, and editing.

\section{Conflict of Interest Statement}

The authors declare that they have no conflict of interest.

\section{References}

Almajan ER, Richter R, Paeger L, Martinelli P, Barth E, Decker T, Larsson N-GOR, Kloppenburg P, Langer T, Rugarli El (2012) AFG3L2 supports mitochondrial protein synthesis and Purkinje cell survival. J Clin Invest 122: 4048-4058. doi:10.1172/jci64604

Amberg-Johnson K, Hari SB, Ganesan SM, Lorenzi HA, Sauer RT, Niles JC, Yeh E (2017) Small molecule inhibition of apicomplexan FtsH1 disrupts plastid biogenesis in human pathogens. Elife 6: pii: e29865. doi:10.7554/elife.29865

Amunts A, Brown A, Toots J, Scheres SHW, Ramakrishnan V (2015) Ribosome. The structure of the human mitochondrial ribosome. Science 348: 95-98. doi:10.1126/science.aaa1193

Anand R, Wai T, Baker MJ, Kladt N, Schauss AC, Rugarli El, Langer T (2014) The i-AAA protease YME1L and OMA1 cleave OPA1 to balance mitochondrial fusion and fission. J Cell Biol 204: 919-929. doi:10.1083/jcb.201308006

Antonicka H, Leary SC, Guercin G-H, Agar JN, Horvath R, Kennaway NG, Harding CO, Jaksch M, Shoubridge EA (2003) Mutations in COX10 result in a defect in mitochondrial heme A biosynthesis and account for multiple, early-onset clinical phenotypes associated with isolated COX deficiency. Hum Mol Genet 12: 2693-2702. doi:10.1093/hmg/ ddg284

Antonicka H, Ostergaard E, Sasarman F, Weraarpachai W, Wibrand F, Pedersen AMB, Rodenburg RJ, van der Knaap MS, Smeitink JAM, Chrzanowska-Lightowlers ZM, et al (2010) Mutations in C12orf65 in patients with encephalomyopathy and a mitochondrial translation defect. Am J Hum Genet 87: 115-122. doi:10.1016/j.ajhg.2010.06.004

Arlt H, Tauer R, Feldmann H, Neupert W, Langer T (1996) The YTA10-12 complex, an AAA protease with chaperone-like activity in the inner membrane of mitochondria. Cell 85: 875-885. doi:10.1016/s0092-8674 (00)81271-4

Auré K, Dubourg O, Jardel C, Clarysse L, Sternberg D, Fournier E, Laforêt P, Streichenberger N, Petiot P, Gervais-Bernard H, et al (2013) Episodic weakness due to mitochondrial DNA MT-ATP6/8 mutations. Neurology 81: 1810-1818. doi:10.1212/01.wnl.0000436067.43384.0b

Baker MJ, Lampe PA, Stojanovski D, Korwitz A, Anand R, Tatsuta T, Langer T (2014) Stress-induced OMA1 activation and autocatalytic turnover regulate OPA1-dependent mitochondrial dynamics. EMBO J 33: 578-593. doi:10.1002/embj.201386474

Belevich I, Joensuu M, Kumar D, Vihinen H, Jokitalo E (2016) Microscopy Image Browser: A Platform for Segmentation and Analysis of Multidimensional Datasets. PLoS Biol 14: e1002340. doi:10.1371/ journal.pbio.1002340
Boulet L, Karpati G, Shoubridge EA (1992) Distribution and threshold expression of the tRNA(Lys) mutation in skeletal muscle of patients with myoclonic epilepsy and ragged-red fibers (MERRF). Am J Hum Genet 51: 1187-1200.

Brown A, Rathore S, Kimanius D, Aibara S, Bai X-C, Rorbach J, Amunts A, Ramakrishnan V (2017) Structures of the human mitochondrial ribosome in native states of assembly. Nat Struct Mol Biol 24: 866-869. doi:10.1038/nsmb.3464

Cagnoli C, Stevanin G, Brussino A, Barberis M, Mancini C, Margolis RL, Holmes SE, Nobili M, Forlani S, Padovan S, et al (2010) Missense mutations in the AFG3L2 proteolytic domain account for $\sim 1.5 \%$ of European autosomal dominant cerebellar ataxias. Hum Mutat 31: 1117-1124. doi:10.1002/humu.21342

Casari G, De Fusco M, Ciarmatori S, Zeviani M, Mora M, Fernandez P, De Michele G, Filla A, Cocozza S, Marconi R, et al (1998) Spastic paraplegia and OXPHOS impairment caused by mutations in paraplegin, a nuclear-encoded mitochondrial metalloprotease. Cell 93: 973-983. doi:10.1016/s0092-8674(00)81203-9

Čižková A, Stránecký V, Mayr JA, Tesařová M, Havliččová V, Paul J, Ivánek R, Kuss AW, Hansíková H, Kaplanová V, et al (2008) TMEM70 mutations cause isolated ATP synthase deficiency and neonatal mitochondrial encephalocardiomyopathy. Nat Genet 40: 1288-1290. doi:10.1038/ ng.246

Davies KM, Anselmi C, Wittig I, Faraldo-Gómez JD, Kühlbrandt W (2012) Structure of the yeast F1Fo-ATP synthase dimer and its role in shaping the mitochondrial cristae. Proc Natl Acad Sci U S A 109: 13602-13607. doi:10.1073/pnas.1204593109

Di Bella D, Lazzaro F, Brusco A, Plumari M, Battaglia G, Pastore A, Finardi A, Cagnoli C, Tempia F, Frontali M, et al (2010) Mutations in the mitochondrial protease gene AFG3L2 cause dominant hereditary ataxia SCA28. Nat Genet 42: 313-321. doi:10.1038/ng.544

Dodt M, Roehr JT, Ahmed R, Dieterich C (2012) FLEXBAR-Flexible Barcode and Adapter Processing for Next-Generation Sequencing Platforms. Biology 1: 895-905. doi:10.3390/biology1030895

Ehses S, Raschke I, Mancuso G, Bernacchia A, Geimer S, Tondera D, Martinou J-C, Westermann B, Rugarli El, Langer T (2009) Regulation of OPA1 processing and mitochondrial fusion by m-AAA protease isoenzymes and OMA1. J Cell Biol 187: 1023-1036. doi:10.1083/ jcb.200906084

Ferreirinha F, Quattrini A, Pirozzi M, Valsecchi V, Dina G, Broccoli V, Auricchio A, Piemonte F, Tozzi G, Gaeta L, et al (2004) Axonal degeneration in paraplegin-deficient mice is associated with abnormal mitochondria and impairment of axonal transport. / Clin Invest 113: 231-242. doi:10.1172/jci20138

Frezza C, Cipolat S, De Brito OM, Micaroni M, Beznoussenko GV, Rudka T, Bartoli D, Polishuck RS, Danial NN, De Strooper B, et al (2006) OPA1 controls apoptotic cristae remodeling independently from mitochondrial fusion. Cell 126: 177-189. doi:10.1016/j.cell.2006.06.025

Friedman JR, Nunnari J (2014) Mitochondrial form and function. Nature 505: 335-343. doi:10.1038/ nature12985

Gorman GS, Pfeffer G, Griffin H, Blakely EL, Kurzawa-Akanbi M, Gabriel J, Sitarz K, Roberts M, Schoser B, Pyle A, et al (2015) Clonal expansion of secondary mitochondrial DNA deletions associated with spinocerebellar ataxia type 28. JAMA Neurol 72: 106-111. doi:10.1001/ jamaneurol.2014.1753

Greber BJ, Bieri P, Leibundgut M, Leitner A, Aebersold R, Boehringer D, Ban N (2015) Ribosome. The complete structure of the 55S mammalian mitochondrial ribosome. Science 348: 303-308. doi:10.1126/science. aаa3872

Harner ME, Unger A-K, Geerts WJ, Mari M, Izawa T, Stenger M, Geimer S, Reggiori F, Westermann B, Neupert W (2016) An evidence based hypothesis on the existence of two pathways of mitochondrial crista formation. Elife 5: 247. doi:10.7554/elife.18853 
Hart T, Chandrashekhar M, Aregger M, Steinhart Z, Brown KR, MacLeod G, Mis M, Zimmermann M, Fradet-Turcotte A, Sun S, et al (2015) HighResolution CRISPR Screens Reveal Fitness Genes and GenotypeSpecific Cancer Liabilities. Cell 163: 1515-1526. doi:10.1016/j. cell.2015.11.015

Head BP, Griparic L, Amiri M, Gandre-Babbe S, Van Der Bliek AM (2009) Inducible proteolytic inactivation of OPA1 mediated by the OMA1 protease in mammalian cells. J Cell Biol 187: 959-966. doi:10.1083/ jcb.200906083

Hornig-Do H-T, Tatsuta T, Buckermann A, Bust M, Kollberg G, Rötig A, Hellmich M, Nijtmans L, Wiesner RJ (2012) Nonsense mutations in the COX1 subunit impair the stability of respiratory chain complexes rather than their assembly. EMBO / 31: 1293-1307. doi:10.1038/ emboj.2011.477

Hudson G, Amati-Bonneau P, Blakely EL, Stewart JD, He L, Schaefer AM, Griffiths PG, Ahlqvist K, Suomalainen A, Reynier P, et al (2008) Mutation of OPA1 causes dominant optic atrophy with external ophthalmoplegia, ataxia, deafness and multiple mitochondrial DNA deletions: a novel disorder of mtDNA maintenance. Brain 131: 329-337. doi:10.1093/brain/awm272

Ishihara N, Fujita Y, Oka T, Mihara K (2006) Regulation of mitochondrial morphology through proteolytic cleavage of OPA1. EMBO I 25: 2966-2977. doi:10.1038/sj.emboj.7601184

Jackson CB, Hahn D, Schröter B, Richter U, Battersby BJ, Schmitt-Mechelke T, Marttinen P, Nuoffer JM, Schaller A (2017) A novel mitochondrial ATP6 frameshift mutation causing isolated complex $V$ deficiency, ataxia and encephalomyopathy. Eur J Med Genet 60: 345-351. doi:10.1016/j. ejmg.2017.04.006

Kardon JR, Yien YY, Huston NC, Branco DS, Hildick-Smith GJ, Rhee KY, Paw BH, Baker TA (2015) Mitochondrial ClpX Activates a Key Enzyme for Heme Biosynthesis and Erythropoiesis. Cell 161: 858-867. doi:10.1016/j. cell.2015.04.017

Keiler KC (2015) Mechanisms of ribosome rescue in bacteria. Nat Rev Microbiol 13: 285-297. doi:10.1038/nrmicro3438

Kondadi AK, Wang S, Montagner S, Kladt N, Korwitz A, Martinelli P, Herholz D, Baker MJ, Schauss AC, Rugarli El (2014) Loss of the m-AAA protease subunit AFG3L2 causes mitochondrial transport defects and tau hyperphosphorylation. EMBO / 33: 1011-1026. doi:10.1002/ embj.201387009

Korwitz A, Merkwirth C, Richter-Dennerlein R, Tröder SE, Sprenger H-G, Quirós PM, López-Otín C, Rugarli El, Langer T (2016) Loss of OMA1 delays neurodegeneration by preventing stress-induced OPA1 processing in mitochondria. J Cell Biol 212: 157-166. doi:10.1083/jcb.201507022

Kremer JR, Mastronarde DN, McIntosh JR (1996) Computer visualization of three-dimensional image data using IMOD. J Struct Biol 116: 71-76. doi:10.1006/jsbi.1996.0013

Li H (2013) Aligning sequence reads, clone sequences and assembly contigs with BWA-MEM. arXiv [q-bio.GN]. Available at: http://arxiv.org/abs/ 1303.3997.

Li H, Durbin R (2010) Fast and accurate long-read alignment with BurrowsWheeler transform. Bioinformatics 26: 589-595. doi:10.1093/ bioinformatics/btp698

Li H, Handsaker B, Wysoker A, Fennell T, Ruan J, Homer N, Marth G, Abecasis G, Durbin R; 1000 Genome Project Data Processing Subgroup (2009) The Sequence Alignment/Map format and SAMtools. Bioinformatics 25: 2078-2079. doi:10.1093/bioinformatics/btp352

Lochmüller H, Johns T, Shoubridge EA (1999) Expression of the E6 and E7 genes of human papillomavirus (HPV16) extends the life span of human myoblasts. Exp Cell Res 248: 186-193. doi:10.1006/excr. 1999.4407

Lykke-Andersen J, Bennett EJ (2014) Protecting the proteome: Eukaryotic cotranslational quality control pathways. J Cell Biol 204: 467-476. doi:10.1083/jcb.201311103
MacVicar T, Langer T (2016) OPA1 processing in cell death and disease: The long and short of it. J Cell Sci 129: 2297-2306. doi:10.1242/jcs.159186

Magner M, Dvorakova V, Tesarova M, Mazurova S, Hansikova H, Zahorec M, Brennerova K, Bzduch V, Spiegel R, Horovitz Y, et al (2015) Erratum to: TMEM70 deficiency: Long-term outcome of 48 patients. J Inherit Metab Dis 38: 583-584. doi:10.1007/s10545-015-9833-9

Mancini C, Hoxha E, Iommarini L, Brussino A, Richter U, Montarolo F, Cagnoli C, Parolisi R, Gondor Morosini DI, Nicolò V, et al (2018) Mice harbouring a SCA28 patient mutation in AFG3L2 develop late-onset ataxia associated with enhanced mitochondrial proteotoxicity. Neurobiol Dis 124: 14-28. doi:10.1016/j.nbd.2018.10.018

Mastronarde DN (1997) Dual-axis tomography: An approach with alignment methods that preserve resolution. J Struct Biol 120: 343-352. doi:10.1006/jsbi.1997.3919

Mastronarde DN (2005) Automated electron microscope tomography using robust prediction of specimen movements. J Struct Biol 152: 36-51. doi:10.1016/j.jsb.2005.07.007

Merkwirth C, Dargazanli S, Tatsuta T, Geimer S, Lower B, Wunderlich FT, von Kleist-Retzow JC, Waisman A, Westermann B, Langer T (2008) Prohibitins control cell proliferation and apoptosis by regulating OPA1-dependent cristae morphogenesis in mitochondria. Genes Dev 22: 476-488. doi:10.1101/gad.460708

Nolden M, Ehses S, Koppen M, Bernacchia A, Rugarli El, Langer T (2005) The m-AAA protease defective in hereditary spastic paraplegia controls ribosome assembly in mitochondria. Cell 123: 277-289. doi:10.1016/j. cell.2005.08.003

Ott M, Amunts A, Brown A (2016) Organization and Regulation of Mitochondrial Protein Synthesis. Annu Rev Biochem 85: 77-101. doi:10.1146/annurev-biochem-060815-014334

Pierson TM, Adams D, Bonn F, Martinelli P, Cherukuri PF, Teer JK, Hansen NF, Cruz P; Mullikin For The Nisc Comparative Sequencing Program JC, Blakesley RW, Golas G, et al (2011) Whole-exome sequencing identifies homozygous AFG3L2 mutations in a spastic ataxia-neuropathy syndrome linked to mitochondrial m-AAA proteases. PLoS Genet 7: e1002325. doi:10.1371/journal.pgen.1002325

Quinlan AR, Hall IM (2010) BEDTools: A flexible suite of utilities for comparing genomic features. Bioinformatics 26: 841-842. doi:10.1093/ bioinformatics/btq033

Quirós PM, Langer T, López-Otín C (2015) New roles for mitochondrial proteases in health, ageing and disease. Nat Rev Mol Cell Biol 16: 345-359. doi:10.1038/nrm3984

Rabl R, Soubannier V, Scholz R, Vogel F, Mendl N, Vasiljev-Neumeyer A, Körner C, Jagasia R, Keil T, Baumeister W, et al (2009) Formation of cristae and crista junctions in mitochondria depends on antagonism between Fcj1 and Su e/g. I Cell Biol 185: 1047-1063. doi:10.1083/jcb.200811099

Rak M, Mcstay GP, Fujikawa M, Yoshida M, Manfredi G, Tzagoloff A (2011) Turnover of ATP synthase subunits in F1-depleted HeLa and yeast cells. FEBS Lett 585: 2582-2586. doi:10.1016/j.febslet.2011.07.011

Richter U, Evans ME, Clark WC, Marttinen P, Shoubridge EA, Suomalainen A, Wredenberg A, Wedell A, Pan T, Battersby BJ (2018) RNA modification landscape of the human mitochondrial tRNALys regulates protein synthesis. Nat Commun 9: 3966. doi:10.1038/s41467-018-06471-z

Richter U, Lahtinen T, Marttinen P, Myöhänen M, Greco D, Cannino G, Jacobs HT, Lietzén N, Nyman TA, Battersby BJ (2013) A mitochondrial ribosomal and RNA decay pathway blocks cell proliferation. Curr Biol 23: 535-541. doi:10.1016/j.cub.2013.02.019

Richter U, Lahtinen T, Marttinen P, Suomi F, Battersby BJ (2015) Quality control of mitochondrial protein synthesis is required for membrane integrity and cell fitness. J Cell Biol 211: 373-389. doi:10.1083/jcb.201504062

Sasarman F, Antonicka H, Shoubridge EA (2008) The A3243G tRNALeu(UUR) MELAS mutation causes amino acid misincorporation and a combined respiratory chain assembly defect partially suppressed by 
overexpression of EFTu and EFG2. Hum Mol Genet 17: 3697-3707. doi:10.1093/hmg/ddn265

Sauer RT, Baker TA (2011) AAA+ Proteases: ATP-Fueled Machines of Protein Destruction. Annu Rev Biochem 80: 587-612. doi:10.1146/annurevbiochem-060408-172623

Schägger H (2006) Tricine-SDS-PAGE. Nat Protoc 1: 16-22. doi:10.1038/ nprot.2006.4

Seneca S, Abramowicz M, Lissens W, Muller MF, Vamos E, de Meirleir L (1996) A mitochondrial DNA microdeletion in a newborn girl with transient lactic acidosis. J Inherit Metab Dis 19: 115-118. doi:10.1007/bf01799407

Steglich G, Neupert W, Langer T (1999) Prohibitins regulate membrane protein degradation by the m-AAA protease in mitochondria. Mol Cell Biol 19: 3435-3442. doi:10.1128/mcb.19.5.3435

Suomalainen A, Battersby BJ (2018) Mitochondrial diseases: the contribution of organelle stress responses to pathology. Nat Rev Mol Cell Biol 19: 77-92. doi:10.1038/nrm.2017.66

Taylor RW, Turnbull DM (2005) Mitochondrial DNA mutations in human disease. Nat Rev Genet 6: 389-402. doi:10.1038/nrg1606

Thorburn DR, Rahman J, Rahman S (2003) Mitochondrial DNA-Associated Leigh Syndrome and NARP. In GeneReviews, Adam MP, Ardinger HH, Pagon RA, Wallace SE, Bean LJH, Mefford HC, Stephens K, Amemiya A, Ledbetter N (eds) Seattle: University of Washington, Seattle

Tyanova S, Temu T, Cox J (2016a) The MaxQuant computational platform for mass spectrometry-based shotgun proteomics. Nat Protoc 11: 2301-2319. doi:10.1038/nprot.2016.136

Tyanova S, Temu T, Sinitcyn P, Carlson A, Hein MY, Geiger T, Mann M, Cox J (2016b) The Perseus computational platform for comprehensive analysis of (prote)omics data. Nat Methods 13: 731-740. doi:10.1038/nmeth.3901
Wai T, Saita S, Nolte H, Müller S, König T, Richter-Dennerlein R, Sprenger HG, Madrenas J, Mühlmeister M, Brandt U, et al (2016) The membrane scaffold SLP2 anchors a proteolytic hub in mitochondria containing PARL and the i-AAA protease YME1L. EMBO Rep 17: 1844-1856. doi:10.15252/embr.201642698

Wilm A, Aw PPK, Bertrand D, Yeo GHT, Ong SH, Wong CH, Khor CC, Petric R, Hibberd ML, Nagarajan N (2012) LoFreq: a sequence-quality aware, ultra-sensitive variant caller for uncovering cell-population heterogeneity from high-throughput sequencing datasets. Nucleic Acids Res 40: 11189-11201. doi:10.1093/ nar/gks918

Yarham JW, Elson JL, Blakely EL, McFarland R, Taylor RW (2010) Mitochondrial tRNA mutations and disease. Wiley Interdiscip Rev RNA 1: 304-324. doi:10.1002/wrna.27

Yoshii SR, Kishi C, Ishihara N, Mizushima N (2011) Parkin mediates proteasome-dependent protein degradation and rupture of the outer mitochondrial membrane. J Biol Chem 286: 19630-19640. doi:10.1074/ jbc.m110.209338

Zhou A, Rohou A, Schep DG, Bason JV, Montgomery MG, Walker JE, Grigorieff N, Rubinstein JL (2015) Structure and conformational states of the bovine mitochondrial ATP synthase by cryo-EM. Elife 4: e10180. doi:10.7554/eLife.10180

Zurita Rendón O, Shoubridge EA (2012) Early complex I assembly defects result in rapid turnover of the ND1 subunit. Hum Mol Genet 21: 3815-3824. doi:10.1093/hmg/dds209

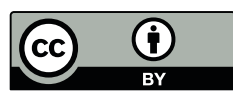

License: This article is available under a Creative Commons License (Attribution 4.0 International, as described at https://creativecommons.org/ licenses/by/4.0/). 